\title{
THE LOGARITHMIC RESIDUE DENSITY OF A GENERALIZED LAPLACIAN
}

\author{
JOUKO MICKELSSON and SYLVIE PAYCHA ${ }^{\bowtie}$ \\ (Received 18 August 2010; accepted 25 October 2010) \\ Communicated by V. Mathai \\ Dedicated to Alan Carey, on the occasion of his 60th birthday
}

\begin{abstract}
We show that the residue density of the logarithm of a generalized Laplacian on a closed manifold defines an invariant polynomial-valued differential form. We express it in terms of a finite sum of residues of classical pseudodifferential symbols. In the case of the square of a Dirac operator, these formulas provide a pedestrian proof of the Atiyah-Singer formula for a pure Dirac operator in four dimensions and for a twisted Dirac operator on a flat space of any dimension. These correspond to special cases of a more general formula by Scott and Zagier. In our approach, which is of perturbative nature, we use either a Campbell-Hausdorff formula derived by Okikiolu or a noncommutative Taylor-type formula.
\end{abstract}

2010 Mathematics subject classification: primary 58J42; secondary 58J40.

Keywords and phrases: residue, index, Dirac operators.

\section{Introduction}

The noncommutative residue on classical pseudodifferential operators introduced by Wodzicki $[14,15]$ has a notable property called locality. That is, it corresponds to a residue density $\operatorname{res}_{x}(A) d x$ integrated over an $n$-dimensional (closed) manifold $M$ :

$$
\begin{aligned}
\operatorname{res}(A) & =\int_{M} \operatorname{res}_{x}(A) d x, \\
\operatorname{res}_{x}(A) d x & :=\frac{1}{(2 \pi)^{n}} \int_{|\xi|=1} \operatorname{tr}\left(\sigma_{-n}(A)(x, \xi)\right) d \xi .
\end{aligned}
$$

Here $\sigma(A)(x, \xi)$ denotes the local symbol of $A$, for $a \in C, \sigma_{a}(A)(x, \xi)$ denotes the homogeneous part of degree $a$ of its symbol, with $(x, \xi)$ varying in the cotangent bundle of $M$, and tr denotes the fibrewise trace. As was observed in [7], this extends to the $\operatorname{logarithm} A=\log Q$ of an elliptic pseudodifferential operator $Q$ of positive order with appropriate spectral cut (we call such an operator admissible).

Exponentiating $\operatorname{res}(\log (Q))$ leads to the residue determinant,

$$
\operatorname{det}_{\text {res }}(Q):=e^{\mathrm{res}(\log Q)},
$$

(C) 2011 Australian Mathematical Publishing Association Inc. 1446-7887/2011 \$16.00 
first introduced by Wodzicki in the case of operators of order zero (see, for example, the survey [3]) and further extended by Scott [12] to elliptic pseudodifferential operators $Q$ with appropriate spectral cuts of positive order. Further logarithmic structures have since been investigated in [10], in relation to topological quantum field theory.

Here, we show that the logarithmic residue density for a generalized Laplacian $Q$,

$$
\operatorname{res}_{x}(\log Q) d x:=\frac{1}{(2 \pi)^{n}}\left[\int_{S_{x}^{*} M} \operatorname{tr}\left(\sigma_{-n}(\log Q)(x, \xi)\right) d \xi\right] d x,
$$

defines an invariant polynomial-valued form in the sense of Weyl (Theorem 3.12). It then follows from Gilkey's invariance theory [2] that this logarithmic residue density can be expressed in terms of Pontryagin and Chern classes.

The presence of a logarithm makes the actual computation of a logarithmic residue density difficult. However, observing that the symbol of a generalized Laplacian reads

$$
\sigma(Q)=|\xi|^{2}+\sigma_{<2}(Q),
$$

where $\sigma_{<2}(Q)$ is of order smaller than two, enables us to carry out computations by means of a noncommutative Taylor-type formula (Theorem 5.1) or a CampbellHausdorff formula (Theorem 4.8), both of which provide ways to compare $\sigma(\log Q)$ with $\log \left(|\xi|^{2}\right)$.

A similar procedure applies, on the operator level, to compute the integrated logarithmic density

$$
\int_{M} \operatorname{res}_{x}(\sigma(\log Q)) d x
$$

in the special case of the square of a twisted Dirac operator $D_{W}$ acting on a twisted $\mathbb{Z}_{2}$-graded spinor bundle $E=S \otimes W$. Indeed, combining the Lichnerowicz formula (7.2) (which compares $D_{W}^{2}$ with a Laplace-Beltrami operator $\Delta^{E}=\left(\nabla^{E}\right)^{*} \nabla^{E}$ built from the underlying connection $\nabla^{E}$ on $E$ ) with a Campbell-Hausdorff formula (which compares $\log D_{W}^{2}$ with $\log \Delta^{E}$ ) yields an expression for the integrated logarithmic superresidue density $\operatorname{sres}\left(\log D_{W}^{2}\right)$ in terms of $\operatorname{sres}\left(\log \left(\Delta^{E}\right)\right)$ and a finite number of superresidues of classical operators involving the curvature of $\nabla^{E}$ (Theorem 7.2). This integrated logarithmic superresidue density turns out to be proportional to the index of the chiral Dirac operator $D_{W}^{+}$(Theorem 6.3):

$$
\operatorname{ind}\left(D^{+}\right)=-\frac{1}{2} \int_{M} \operatorname{sres}_{x}\left(\log D_{W}^{2}\right) d x .
$$

This was observed independently by Scott in [12] and the second author in some unpublished lecture notes delivered at the University of Los Andes, Colombia. Thus, locality in the Atiyah-Singer index theorem is closely related to the local properties of the noncommutative residue.

We compute the index in two concrete examples. Our first example is for a twisted Dirac operator on a flat space (Theorem 8.1), along the lines described above using a Campbell-Hausdorff formula, and then for a pure Dirac operator in 
four dimensions, using a Taylor-type formula. For the second example, we first derive simple formulas (see Proposition 9.3) for (super)residues of certain expressions involving the derivatives of the Christoffel symbols, which can then be used to derive the index in four dimensions. In this way, we recover the Atiyah-Singer index theorem for a pure Dirac operator on a four-dimensional spin manifold.

With the perturbative approach adopted here, using either a Campbell-Hausdorff or a noncommutative Taylor formula, we were, unfortunately, unable to derive the general Atiyah-Singer formula due to Scott and Zagier announced in [10] and proven in [11]. This perturbative approach, nevertheless, provides a pedestrian proof in the cases investigated here, and useful intermediate results such as Theorem 3.12 and Theorem 5.1, which we feel are of interest in their own right.

\section{Notation}

Given a real, oriented Euclidean vector space $V$ of even dimension $n=2 p$, there is a unique $\mathbb{Z}_{2}$-graded complex Clifford module $S=S^{+} \oplus S^{-}$, the spinor module, such that the complex Clifford algebra $C(V) \otimes \mathbb{C}$ may be identified with $\operatorname{End}(S)$ and $\operatorname{dim}(S)=2^{p}$. An auxiliary linear complex space $W$ yields a $\mathbb{Z}_{2}$-graded twisted Clifford module $E=S \otimes W$.

Let

$$
\begin{aligned}
c: \Lambda V & \rightarrow C(V) \\
e_{i_{1}} \wedge \cdots \wedge e_{i k} & \mapsto c\left(e_{i_{1}}\right) \cdots c\left(e_{i_{k}}\right)
\end{aligned}
$$

be the quantization map. To simplify our notation, we set $\gamma_{j}=c\left(e_{j}\right)$, so that the grading operator reads

$$
\Gamma=i^{p} \gamma_{1} \cdots \gamma_{n} \text {. }
$$

Notice that $\Gamma^{2}=\mathrm{Id}$. The cyclicity of the trace, combined with the Clifford relations, implies that the supertrace $\operatorname{str}:=\operatorname{tr} \circ \Gamma$ on $\operatorname{End}(E)$ satisfies the following property for a matrix $M \in \operatorname{End}(W)$ viewed as an element of $\operatorname{End}(E)$ :

$$
\operatorname{str}\left(M \gamma_{i_{1}} \cdots \gamma_{i_{k}}\right)=0 \quad \text { if } k<n, \quad \operatorname{str}\left(M \gamma_{1} \cdots \gamma_{n}\right)=(-2 i)^{p} \operatorname{tr}(M),
$$

since $\operatorname{dim} \operatorname{End}(\mathcal{S})=2^{p}$. On the other hand, setting

$$
\sigma_{i j}=\frac{1}{8}\left[\gamma_{i}, \gamma_{j}\right]=\frac{1}{4} \gamma_{i} \gamma_{j} \quad \text { if } i \neq j,
$$

we have that, for any permutation $\tau \in \Sigma_{n}$ with signature $|\tau|$,

$$
\begin{aligned}
\operatorname{str}\left(\sigma_{\tau(1) \tau(2)} \sigma_{\tau(3) \tau(4)} \cdots \sigma_{\tau(n-1) \tau(n)}\right) & =\frac{(-1)^{|\tau|}}{4^{p}} \operatorname{str}\left(\gamma_{1} \cdots \gamma_{n}\right) \\
& =\frac{(-1)^{|\tau|}(-i)^{p}}{2^{p}} .
\end{aligned}
$$

These constructions carry over to bundles, for which we abusively use the same notation.

Let

$$
E=S \otimes W=E^{+} \oplus E^{-},
$$


with $E^{+}=S^{+} \otimes W, E^{-}=S^{-} \otimes W$, be a twisted $\mathbb{Z}_{2}$-graded spinor bundle over an even $n=2 p$-dimensional closed Riemannian manifold $M$ whose auxiliary bundle $W$ is equipped with a connection $\nabla^{W}$. Let $F$ be a vector bundle over $M$, and let $C \ell(M, F)$ denote the algebra of classical pseudodifferential operators acting on the space $C^{\infty}(M, F)$ of smooth sections of the vector bundle $F$.

Let

$$
D=\sum_{i=1}^{n} c\left(e_{i}\right) \nabla_{e_{i}}^{S} \in C \ell(M, S)
$$

be the Dirac operator, where $\nabla^{S}$ is the spinor connection, $c$ stands for Clifford multiplication and $\left\{e_{i}: i=1, \ldots, n\right\}$ is an orthonormal tangent frame on $M$. In local coordinates we shall also write $\gamma_{i}$ for $c\left(e_{i}\right)$.

Let

$$
\nabla^{E}:=\nabla^{S} \otimes 1+1 \otimes \nabla^{W}
$$

be a connection on the twisted bundle $E=S \otimes W$, and let

$$
D_{W}=\sum_{i=1}^{n} c\left(e_{i}\right) \nabla_{e_{i}}^{E} \in C \ell(M, E)
$$

be the corresponding twisted Dirac operator. The chiral Dirac operator $D_{W}^{+}$and its formal adjoint $D_{W}^{-}$act from $C^{\infty}\left(M, E^{+}\right)$to $C^{\infty}\left(M, E^{-}\right)$, and conversely.

\section{The logarithmic residue density as an invariant polynomial}

Following Gilkey's notation (see [2, (2.4.3)]), for a multi-index $\alpha=\left(\alpha_{1} \ldots \alpha_{s}\right)$, we introduce formal variables $g_{i j / \alpha}=\partial_{\alpha} g_{i j}$ for the partial derivatives of the metric tensor $g$ on $M$ and the connection $\omega$ on the external bundle. Let us set

$$
\operatorname{ord}\left(g_{i j / \alpha}\right)=|\alpha|=\alpha_{1}+\cdots+\alpha_{s} ; \quad \operatorname{ord}\left(\omega_{i / \beta}\right)=|\beta| .
$$

Inspired by Gilkey (see [2, (1.8.18) and (1.8.19)]), we give the following definition.

DEFINITION 3.1. We call a classical operator $A \in C \ell(M, E)$ of order a geometric if, in any local trivialization, the homogeneous components $\sigma_{a-j}(A)$ are homogeneous of order $j$ in the jets of the metric and of the connection.

REMARK 3.2. A differential operator

$$
A=\sum_{|\alpha| \leq a} c_{\alpha}(x) \partial_{x}^{\alpha} \in C \ell(M, E)
$$

is geometric if $c_{\alpha}(x)$ is homogeneous of order $j=a-|\alpha|$ in the jets of the metric and of the connection $\nabla^{W}$. Here we use the standard notation $\partial_{x}{ }^{\alpha}=\partial_{\alpha_{1}} \ldots \partial_{\alpha_{s}}$. 
EXAMPLE 3.3. The Laplace-Beltrami operator

$$
\Delta_{g}=-\frac{1}{\sqrt{g}} \sum_{i=1, j=1}^{n} \partial_{i}\left(\sqrt{g} g^{i j} \partial_{j}\right)
$$

is geometric.

More generally, [2, (2.4.22)] shows that

$$
\Delta_{p}=d_{p-1} \delta_{p-1}+\delta_{p} d_{p}
$$

on $p$-forms, where

$$
\delta_{k}=(-1)^{n k+1} \star_{n-k} d_{n-k-1} \star_{k+1},
$$

is a geometric operator. Indeed, each derivative applied to $\star$ reduces the order of differentiation by one and increases the order in the jets of the metric by one.

EXAMPLE 3.4. The square of the twisted Dirac operator

$$
D_{W}^{2}=-\sum_{i j} g^{i j}\left(\nabla_{i}^{E} \nabla_{j}^{E}+\sum_{k} \Gamma_{i j}^{k} \nabla_{k}^{E}\right)+\sum_{i<j} c\left(d x^{i}\right) c\left(d x^{j}\right)\left[\nabla_{i}^{E}, \nabla_{j}^{E}\right]
$$

is a geometric operator.

Geometric operators form an algebra.

LEMMA 3.5. The product of two geometric operators $A$ and $B$ in $C \ell(M, E)$ is again a geometric operator.

PROOF. Since the product $A B$ has symbol

$$
\sigma(A B) \sim \sum_{\alpha} \frac{(-i)^{|\alpha|}}{\alpha !} \partial_{\xi}^{\alpha} \sigma(A) \partial_{x}^{\alpha} \sigma(B)
$$

we have

$$
\sigma_{a+b-k}(A B)=\sum_{|\alpha|+i+j=k} \frac{(-i)^{|\alpha|}}{\alpha !} \partial_{\xi}^{\alpha} \sigma_{a-i}(A) \partial_{x}^{\alpha} \sigma_{b-j}(B)
$$

where $a$ is the order of $A$ and $b$ is the order of $B$ and where $\sigma(C)$ denotes the symbol of the operator $C$. Thus, if the homogeneous components $\sigma_{a-i}(A)$ and $\sigma_{b-j}(B)$ have degree $i$ and $j$, respectively, in the jets of the metric and of the connection, the homogeneous component $\sigma_{a+b-k}(A B)$ is homogeneous of degree $i+j+|\alpha|=k$.

Following [1], we call a second order differential operator acting on $C^{\infty}(M, E)$ with leading symbol $|\xi|^{2}$ a generalized Laplacian on $E$. Since generalized Laplacians are expected to be geometric (see the examples in Section 1), we assume that generalized Laplacians are geometric without further specification. Note that a generalized Laplacian is admissible (see Appendix A). The following result provides a way to build families of geometric operators. See Appendix A for details on spectral cuts.

Proposition 3.6. Let $Q \in C \ell(M, E)$ be a generalized Laplacian with spectral cut $\theta$. Then, for any geometric operator $A \in C \ell(M, E)$, the family $A(z):=A Q_{\theta}^{z}$ is a family of geometric operators. 
Proof. By Lemma 3.5, it is sufficient to prove the result for $A=I$. For convenience, we drop the explicit mention of the spectral cut.

Since

$$
Q^{z}=\frac{1}{2 i \pi} \int_{\Gamma} \lambda^{z}(Q-\lambda)^{-1} d \lambda,
$$

where $\Gamma$ is a contour described in Appendix A (see formula (A.2)), we need to investigate the resolvent $R(Q, \lambda)=(Q-\lambda)^{-1}$, the homogeneous components $\sigma_{2-j}(R(Q, \lambda))$ of the symbol of which are defined inductively on $j$ by

$$
\begin{aligned}
\sigma_{-2}(R(Q, \lambda)) & =\left(\sigma_{2}(Q)\right)^{-1} \\
\sigma_{-2-j}(R(Q, \lambda)) & =-\sigma_{-2}(R(Q, \lambda)) \\
& \times \sum_{k+l+|\alpha|=j, l<j} \frac{(-i)^{|\alpha|}}{\alpha !} D_{\xi}^{\alpha} \sigma_{2-k}(Q) D_{x}^{\alpha} \sigma_{-2-l}(R(Q, \lambda)) .
\end{aligned}
$$

Using (3.1), one shows, by induction on $j$, that $\sigma_{-2-j}(R(Q, \lambda))$ is a finite sum of expressions of the type

$$
(-i)^{|\alpha|}\left(|\xi|^{2}-\lambda\right)^{-1-k} D_{\xi}^{\alpha_{1}} D_{x}^{\beta_{1}} \sigma_{2-l_{1}}(Q) \cdots D_{\xi}^{\alpha_{k}} D_{x}^{\beta_{k}} \sigma_{2-l_{k}}(Q),
$$

with $|l|+|\alpha|=j,|\alpha|=|\beta|$. Substituting this into

$$
\sigma_{2 z-j}\left(Q^{z}\right)(x, \xi)=-\frac{1}{2 i \pi} \int_{\Gamma} \lambda^{z} \sigma_{-2-j}(R(Q, \lambda))(x, \xi) d \lambda,
$$

and applying repeated integrations by parts to compute the Cauchy integrals,

$$
\frac{1}{2 i \pi} \int_{\Gamma} \lambda^{z}\left(|\xi|^{2}-\lambda\right)^{-k-1} d \lambda=(-1)^{k} \frac{z(z+1) \cdots(z+(k-1))}{k !}|\xi|^{2(z-k)}
$$

leads to a combination of symbols of the type

$$
|\xi|^{q(z-k)} D_{\xi}^{\alpha_{1}} D_{x}^{\beta_{1}} \sigma_{q-l_{1}}(Q)(x, \xi) \cdots D_{\xi}^{\alpha_{k}} D_{x}^{\beta_{k}} \sigma_{q-l_{k}}(Q)(x, \xi),
$$

with $|l|+|\alpha|=j$ and $|\alpha|=|\beta|$. Since $\sigma_{2-l}(Q)$ is homogeneous of order $l$ in the jets of the metric and of the connection, it follows that, for any complex number $z$, the symbol $\sigma_{2 z-j}\left(Q^{z}\right)$ is homogeneous of order $j$ as it is a linear combination of products of homogeneous expressions of order $j_{i}$ in the jets of the metric and of the connection such that $j_{1}+\cdots+j_{k}=j$.

The notion of a geometric operator extends to logarithms of admissible operators as defined in Appendix A.

Definition 3.7. We say that the $\log$ arithm $\log _{\theta} A$ (see formula (A.4) in Appendix A) of an admissible operator $A \in C \ell(M, E)$ of order $a$ with spectral cut $\theta$ is geometric if, in any local trivialization, the homogeneous components $\sigma_{-j, 0}\left(\log _{\theta} A\right.$ ) (see Appendix A) are homogeneous of order $j$ in the jets of the metric and of the connection. 
REMARK 3.8. This can be generalized to any log-polyhomogeneous operator $A$ of order $a$ and logarithmic degree $k$, by requiring that all the coefficients $\sigma_{a-j, l}(A)$ (see Appendix A) for $l \in\{0, \ldots, k\}$ in the logarithmic expansion of the symbol are homogeneous of order $a-j$ in the jets of the metric and of the connection.

COROLLARY 3.9. The logarithm of a generalized Laplacian is a geometric operator.

PROOF. Again, we drop the explicit mention of the spectral cut. Differentiating (3.3) with respect to $z$ at zero shows that $\sigma_{-j, 0}(\log Q)(x, \xi)$ is a linear combination of symbols of the type

$$
|\xi|^{-2 k} D_{\xi}^{\alpha_{1}} D_{x}^{\beta_{1}} \sigma_{2-l_{1}}(Q)(x, \xi) \cdots D_{\xi}^{\alpha_{k}} D_{x}^{\beta_{k}} \sigma_{2-l_{k}}(Q)(x, \xi),
$$

with $|l|+|\alpha|=j$, and $|\alpha|=|\beta|$. Hence, the symbol $\sigma_{-j, 0}(\log Q)$ is homogeneous of order $j$ as it is a linear combination of products of homogeneous expressions of order $j_{i}$ (with $j_{1}+\cdots+j_{k}=j$ ) in the jets of the metric and of the connection.

REMARK 3.10. This is a particular instance of a more general result, namely that the derivative $A^{\prime}(0)$ at zero of a holomorphic germ $A(z) \in C \ell(M, E)$ of geometric operators around zero is also geometric. This follows from the expression (see, for example, [9])

$$
\left(\sigma\left(A^{\prime}(z)\right)\right)_{\alpha(z)-j}=\partial_{z}\left(\sigma_{\alpha(z)-j}(A(z))\right)
$$

of the homogeneous components of the symbol of the derivative $A^{\prime}(0)$ in terms of the derivative of the homogeneous components of the symbol of $A(z)$. Here $\alpha(z)$ is the order of $A(z)$.

Adopting Gilkey's notation (see [2, Section 2.4]) let us denote by $\mathcal{P}_{n, k, p}^{g, \nabla^{W}}$ (which we write $\mathcal{P}_{n, k, p}^{g}$ if $E=S$ ) the linear space consisting of $p$-form-valued invariant polynomials that are homogeneous of order $k$ in the jets of the metric and of the connection $\nabla^{W}$. Note that, by invariant, we mean that the polynomials agree in any coordinate system around $x_{0}$ which is normalized with respect to the point $x_{0}$, that is, such that $g_{i j}\left(x_{0}\right)=\delta_{i-j}$ and $\partial_{k} g_{i j}\left(x_{0}\right)=0$. Also, the order in the jets of the metric is defined to be ord $\left(\partial_{x}^{\alpha} g_{i j}\right)=|\alpha|$.

EXAMPLE 3.11. The scalar curvature $r_{M}$ belongs to $\mathcal{P}_{n, 2,0}^{g}$ since it reads

$$
r_{M}=2 \sum_{i, j}\left(\partial_{i, j}^{2} g_{i j}-\partial_{i, i}^{2} g_{j j}\right)
$$

in the Riemannian normal coordinate system.

THEOREM 3.12. The logarithmic residue density of a generalized Laplacian $Q$ on $E$,

$$
\begin{aligned}
R_{n}(x, Q) & :=\operatorname{res}_{x}\left(\log _{\theta} Q\right) d x \\
& :=\frac{1}{(2 \pi)^{n}}\left[\int_{S_{x}^{*} M} \operatorname{tr}\left(\sigma_{-n, 0}\left(\log _{\theta} Q\right)(x, \xi)\right) d \xi\right] d x,
\end{aligned}
$$


is an invariant polynomial in $\mathcal{P}_{n, n, n}^{g, \nabla^{W}}$, and $R_{n}(x, Q)$ is therefore expressed in terms of Pontryagin forms of the tangent bundle and Chern forms on the auxiliary bundle.

PROOF. By Proposition 3.6 the logarithm (we drop the spectral cut) $\log Q$ is geometric, so that $\sigma_{-n}(\log Q)(x, \xi)$ is homogeneous of degree $n$ in the jets of the metric and of the connection. Integrating this expression in $\xi$ on the unit cosphere shows that the residue density lies in $\mathcal{P}_{n, n, n}^{g, \nabla^{W}}$.

The result follows since $\mathcal{P}_{n, n, n}^{g, \nabla^{W}}$ is generated by Pontryagin forms on the tangent bundle (see [2, Theorem 2.6.2]) and Chern forms on the auxiliary bundle.

REMARK 3.13. The logarithmic residue density is clearly additive on direct sums $E_{1} \oplus E_{2}$ of vector bundles over a closed manifold $M$,

$$
R_{n}\left(x, Q_{1} \oplus Q_{2}\right)=R_{n}\left(x, Q_{1}\right)+R_{n}\left(x, Q_{2}\right),
$$

but there is, a priori, no reason why it should be multiplicative on tensor products $E_{1} \otimes E_{2} \rightarrow M_{1} \times M_{2}$ of vector bundles $E_{i}$ over closed manifolds $M_{i}$.

\section{The logarithmic residue density via the Campbell-Hausdorff formula}

The Campbell-Hausdorff formula provides a first approach to computing a local logarithmic residue density. By the results of Okikiolu [6], for two admissible classical pseudodifferential operators with scalar leading symbols $A$ and $B$ in $C \ell(M, E)$, and, under suitable technical assumptions on their spectrum to ensure that their logarithms are well defined,

$$
\log (A B) \sim \log A+\log B+\sum_{k=2}^{\infty} C^{(k)}(\log A, \log B) .
$$

Here, $C^{(k)}(\log A, \log B)$ are Lie monomials given by

$$
C^{(k)}(P, Q):=\sum_{j=1}^{\infty} \frac{(-1)^{j+1}}{(j+1)} \sum \frac{\left(\operatorname{Ad}_{P}\right)^{\alpha_{1}}\left(\operatorname{Ad}_{Q}\right)^{\beta_{1}} \cdots\left(\operatorname{Ad}_{P}\right)^{\alpha_{j}}\left(\operatorname{Ad}_{Q}\right)^{\beta_{j}}}{\left(1+\sum_{l=1}^{j} \beta_{l}\right) \alpha_{1} ! \cdots \alpha_{j} ! \beta_{1} ! \cdots \beta_{j} !}(Q),
$$

which vanish if $\beta_{j}>1$, or if $\beta_{j}=0$ and $\alpha_{j}>1$, and where the inner sum runs over $j$-tuples of pairs $\left(\alpha_{i}, \beta_{i}\right)$ such that $\alpha_{i}+\beta_{i}>0$ and $\sum_{i=1}^{j} \alpha_{i}+\beta_{i}=k$. In formula (4.2), $\operatorname{Ad}_{P}(Q)=[P, Q]$ and the symbol $\sim$ means that, for any integer $N$, the difference

$$
F_{N}(A, B):=\log (A B)-\log A-\log B-\sum_{k=2}^{n+1} C^{(k)}(\log A, \log B)
$$

is of order smaller than $-N$. The fact that the leading symbols are scalar ensures that the order of $C^{(k)}(\log A, \log B)$ decreases as $k$ increases. Hence, it provides a good control on the asymptotics since the adjoint operations $\operatorname{ad}_{\log A}$ and $\operatorname{ad}_{\log B}$ decrease the order by one unit. 
Proposition 4.1 (See [6]). Let $A, B \in C \ell(M, E)$ be invertible elliptic operators with scalar leading symbols such that $A, B$ and their product $A B$ have well-defined logarithms. Then $F_{n}(A, B)$, defined as in (4.3), where $n$ is the dimension of the underlying manifold $M$, is a trace-class operator and hence its Wodzicki residue vanishes. Both its trace and its residue vanish (see also [12]),

$$
\operatorname{res}(\log (A B)-\log A-\log B)=0 .
$$

The proof in [6] is based on an expansion on the level of symbols which we now describe for future use. We consider the algebra $\mathcal{F} \mathcal{S}(U)$ of formal symbols on an open subset $U$ of $\mathbb{R}^{n}$, equipped with the symbol product $\star$ given by

$$
\sigma_{1} \star \sigma_{2}(x, \xi)=\sum_{\alpha \in \mathbb{N}^{n}} \frac{(-i)^{|\alpha|}}{\alpha !} \partial_{\xi}^{\alpha} \sigma_{1}(x, \xi) \partial_{x}^{\alpha} \sigma_{2}(x, \xi) .
$$

Let $\{\sigma, \tau\}_{\star}:=\sigma \star \tau-\tau \star \sigma$ denote the associated star bracket.

EXAMPLE 4.2. If $\sigma$ is polynomial, then this formal power series of symbols with decreasing order becomes a finite sum, as in the following example which is of interest to us:

$$
\left\{|\xi|^{2}, \tau\right\}_{\star}=\left(L_{x}+\Delta_{x}\right) \tau
$$

where we define

$$
L_{x}:=-2 i \sum_{a=1}^{n} \xi_{a} \partial_{x_{a}} \quad \text { and } \quad \Delta_{x}:=-\sum_{a=1}^{n} \partial_{x_{a}}^{2} .
$$

We define $\operatorname{ad}_{\sigma}^{* k}$ by induction on $k$, setting $\operatorname{ad}_{\sigma}^{* 0}(\tau)=\tau$ and $\operatorname{ad}_{\sigma}^{*(k+1)}(\tau):=$ $\left\{\sigma, \operatorname{ad}_{\sigma}^{* k}(\tau)\right\}$.

EXAMPLE 4.3. $\operatorname{ad}_{|\xi|^{2}}^{* k}(\tau)=\left(L_{x}+\Delta_{x}\right)^{k} \tau$ is a symbol of order $\operatorname{ord}(\tau)+k$.

Here is another example of interest to us.

EXAMPLE 4.4.

$$
\begin{aligned}
\left\{\log |\xi|^{2}, \tau\right\}_{\star}= & \sum_{|\alpha|=0}^{\infty} \frac{(-i)^{|\alpha|}}{\alpha !} \partial_{\xi}^{\alpha} \log |\xi|^{2} \partial_{x}^{\alpha} \tau(x, \xi) \\
= & -2 i \sum_{j=1}^{n} \frac{\xi_{j}}{|\xi|^{2}} \partial_{x_{j}} \tau(x, \xi)-\sum_{i=1}^{n} \frac{1}{|\xi|^{2}} \partial_{x_{i}}^{2} \tau(x, \xi) \\
& +2 \sum_{i, j=1}^{n} \frac{\xi_{i}, \xi_{j}}{|\xi|^{4}} \partial_{x_{i} x_{j}}^{2} \tau(x, \xi)+\cdots
\end{aligned}
$$

We now specialize to the algebra $\mathcal{F} \mathcal{S}_{\mathrm{cl}}(U)$ of polyhomogeneous formal symbols. The resolvent of a polyhomogeneous formal symbol $\sigma$ of order $a$,

$$
r_{\star}(\sigma, \lambda)=(\lambda-\sigma)^{\star^{-1}},
$$


as a solution to $(\lambda-\sigma) \star r=1$, has homogeneous components $\sigma_{a-j}\left(r_{\star}(\sigma, \lambda)\right)$ of degree $a-j$ in $\left(\xi, \lambda^{1 / a}\right)$ defined, inductively on $j$, by

$$
\begin{aligned}
& \sigma_{-a}\left(r_{\star}(\sigma, \lambda)\right)=\left(\sigma_{a}-\lambda\right)^{-1}, \\
&=\sigma_{-a-j}\left(r_{\star}(\sigma, \lambda)\right)=-\sigma_{-a}\left(r_{\star}(\sigma, \lambda)\right) \\
& \times \sum_{k+l+|\alpha|=j, l<j} \frac{(-i)^{|\alpha|}}{\alpha !} D_{\xi}^{\alpha} \sigma_{a-k}(\sigma) D_{x}^{\alpha} \sigma_{-a-l}\left(r_{\star}(\sigma, \lambda)\right) .
\end{aligned}
$$

DEFINITION 4.5. We call a formal symbol $\sigma$ in $\mathcal{F S}_{\mathrm{cl}}(U)$ admissible with spectral cut $\theta$ if, for every $(x, \xi) \in T^{*} U-\{0\}$, the leading symbol matrix $\sigma^{L}(x, \xi)$ has no eigenvalue in a conical neighbourhood of the ray $L_{\theta}=\left\{r e^{i \theta}: r \geq 0\right\}$. In particular, such a symbol is elliptic.

The logarithm of an admissible formal polyhomogeneous symbol $\sigma$ is defined by (see, for example, [6]):

$$
\log _{\star}(\sigma):=\frac{i}{2 \pi}\left(\partial_{z} \int_{\Gamma} \lambda^{z}(\lambda-\sigma)^{\star^{-1}} d \lambda\right)_{\left.\right|_{z=0}},
$$

for a contour $\Gamma$ which encloses the eigenvalues of the leading symbol of $\sigma$. The Campbell-Hausdorff formula for admissible formal polyhomogeneous symbols $\sigma$ and $\tau$ with scalar leading symbols reads (see [6, Lemma 2.7]):

$$
\log _{\star}(\sigma \star \tau) \sim \log _{\star} \sigma+\log _{\star} \tau+\sum_{k=2}^{\infty} C_{\star}^{(k)}\left(\log _{\star} \sigma, \log _{\star} \tau\right),
$$

where $C_{\star}^{(k)}\left(\log _{\star} \sigma, \log _{\star} \tau\right)$ are Lie monomials defined as in (4.2), and $\operatorname{ad}_{P}(Q)$ is replaced by

$$
\operatorname{ad}_{p}^{\star}(q):=\{p, q\}_{\star}:=p \star q-q \star p .
$$

The beginning of the expansion in equation (4.1) reads:

$$
\begin{aligned}
\log _{\star}(\sigma \star \tau) \sim \log _{\star} \sigma & +\log _{\star} \tau+\frac{1}{2}\left\{\log _{\star} \sigma, \log _{\star} \tau\right\}_{\star} \\
& +\frac{1}{12}\left\{\log _{\star} \sigma,\left\{\log _{\star} \sigma, \log _{\star} \tau\right\}_{\star}\right\}_{\star} \\
& -\frac{1}{12}\left\{\log _{\star} \tau,\left\{\log _{\star} \sigma, \log _{\star} \tau\right\}_{\star}\right\}_{\star} \\
& -\frac{1}{24}\left\{\log _{\star} \tau,\left\{\log _{\star} \sigma,\left\{\log _{\star} \sigma, \log _{\star} \tau\right\}_{\star}\right\}_{\star}\right\}_{\star} \cdots .
\end{aligned}
$$

REMARK 4.6. If $\tau$ is classical, then $C_{\star}^{(k)}\left(\log |\xi|^{2}, \tau\right)$ is classical since the bracket $\left\{\log |\xi|^{2}, \sigma\right\}_{\star}$ with a classical symbol $\sigma$ is classical.

REMARK 4.7. If $\tau$ has negative order, then the order $\alpha_{k}$ of $C_{\star}^{(k)}\left(\log |\xi|^{2}, \tau\right)$ is negative and decreases with $k$. Indeed, $\alpha_{k+1}$ corresponds either to the order of

$$
\left\{\log |\xi|^{2}, C_{\star}^{(k)}\left(\log |\xi|^{2}, \tau\right)\right\}_{\star},
$$

which, by (4.6), is $\alpha_{k}-1$, or to the order of $\left\{\tau, C_{\star}^{(k)}\left(\log |\xi|^{2}, \tau\right)\right\}_{\star}$, which is $\operatorname{ord}(\tau)+$ $\alpha_{k}$ and, hence, smaller than $\alpha_{k}$. 
THEOREM 4.8. The logarithmic residue density (3.4) of a generalized Laplacian $Q$ on $E$ is a finite sum of residue densities of classical symbols:

$$
\begin{aligned}
\operatorname{res}_{x}(\log Q)= & \operatorname{res}_{x}\left(\log _{\star}\left(|\xi|^{-2} \star \sigma(Q)(x, \xi)\right)\right)+\sum_{j=1}^{n} \frac{(-1)^{j}}{(j+1) !} \\
& \times \sum_{k=2}^{n} \operatorname{res}_{x}\left(C_{\star}^{(k)}\left(\log |\xi|^{2},\left(|\xi|^{-2} \star \sigma_{<2}(Q)(x, \xi)\right)^{*(j+1)}\right)\right), \\
= & \sum_{j=1}^{n} \frac{(-1)^{j}}{(j+1) !} \operatorname{res}_{x}\left(\left(|\xi|^{-2} \star \sigma_{<2}(Q)(x, \xi)\right)^{*(j+1)}\right) \\
& +\sum_{j=1}^{n} \frac{(-1)^{j}}{(j+1) !} \\
& \times \sum_{k=2}^{n} \operatorname{res}_{x}\left(C_{\star}^{(k)}\left(\log |\xi|^{2},\left(|\xi|^{-2} \star \sigma_{<2}(Q)(x, \xi)\right)^{*(j+1)}\right)\right),
\end{aligned}
$$

where we have set

$$
\sigma(Q)(x, \xi)=|\xi|^{2}+\sigma_{<2}(Q)(x, \xi) .
$$

PROOF. We write

$$
\sigma(Q)(x, \xi)=|\xi|^{2} \star\left(1+|\xi|^{-2} \star \sigma_{<2}(Q)(x, \xi)\right) .
$$

Applying the Campbell-Hausdorff formula (4.9) to $\sigma=|\xi|^{2}$ and

$$
\tau=1+|\xi|^{-2} \star \sigma_{<2}(Q)
$$

yields

$$
\begin{aligned}
\sigma(\log Q)(x, \xi) \sim & \log _{\star} \sigma(Q)(x, \xi) \\
\sim & 2 \log |\xi|+\log _{\star}\left(|\xi|^{-2} \star \sigma(Q)(x, \xi)\right) \\
& \quad+\sum_{k=2}^{\infty} C_{\star}^{(k)}\left(\log |\xi|^{2}, \log _{\star}\left(1+|\xi|^{-2} \star \sigma_{<2}(Q)\right)\right) \\
& \sim 2 \log |\xi|+\sum_{j=1}^{n} \frac{(-1)^{j}}{(j+1) !}\left(|\xi|^{-2} \star \sigma_{<2}(Q)(x, \xi)\right)^{*(j+1)} \\
& +\sum_{k=2}^{\infty} C_{\star}^{(k)}\left(\log |\xi|^{2}, \log _{\star}\left(1+|\xi|^{-2} \star \sigma_{<2}(Q)\right)\right) .
\end{aligned}
$$

This shows that

$$
\log _{\star} \sigma(Q)-\log |\xi|^{2}
$$

is a classical symbol since the logarithm,

$$
\log _{\star} \tau \sim \sum_{j=1}^{\infty} \frac{(-1)^{j}}{(j+1) !}\left(|\xi|^{-2} \star \sigma_{<2}(Q)(x, \xi)\right)^{*(j+1)},
$$

is classical and, hence, the corresponding Lie monomials are classical by Remark 4.6. 
Applying Remark 4.7 to

$$
\tau=\log _{\star}\left(1+|\xi|^{-2} \star \sigma_{<2}(Q)\right),
$$

which has negative order, shows that

$$
C_{\star}^{(k)}\left(\log |\xi|^{2}, \log _{\star}\left(1+|\xi|^{-2} \star \sigma_{<2}(Q)\right)\right)
$$

has order smaller than $-k$. Since the residue vanishes on symbols of order smaller than $-n$, and

$$
\left(|\xi|^{-2} \star \sigma_{<2}(Q)(x, \xi)\right)^{*(j+1)}
$$

has order no larger than $-(j+1)$, implementing the residue yields

$$
\begin{aligned}
\operatorname{res}_{x}(\log Q)=\operatorname{res}_{x}\left(\log _{\star}\left(|\xi|^{-2} \star \sigma(Q)(x, \xi)\right)\right) & \\
& +\sum_{k=2}^{n} \operatorname{res}_{x}\left(C_{\star}^{(k)}\left(\log |\xi|^{2} \star \log _{\star}\left(1+|\xi|^{-2} \star \sigma_{<2}(Q)\right)\right)\right) .
\end{aligned}
$$

Replacing

$$
\log _{\star}\left(|\xi|^{-2} \star \sigma(Q)(x, \xi)\right)=\log _{\star}\left(1+|\xi|^{-2} \star \sigma_{<2}(Q)\right)
$$

by its expansion yields the result.

\section{The logarithmic residue density via a noncommutative Taylor expansion}

A noncommutative Taylor-type formula provides an alternative way to express logarithmic residue densities. We extend the formulas for noncommutative Taylor expansions derived in [8] to formal polyhomogeneous symbols.

Given an analytic function,

$$
\phi(z)=\phi_{0}+\phi_{1} z+\phi_{2} z^{2}+\cdots,
$$

and an admissible symbol $\sigma$ in $\mathcal{F S}_{\mathrm{cl}}(U)$, we write

$$
\Phi_{\star}(\sigma)=\frac{1}{2 i \pi} \int_{\Gamma} r_{\star}(\lambda, \sigma) \phi(\lambda) d \lambda,
$$

where the resolvent, $r_{\star}(\lambda, \sigma)$, is defined by (3.1) and $\Gamma$ is a contour which encloses the eigenvalues of the leading symbol of $\sigma$. Applying this to the higher derivative, $\phi^{(k)}$, yields

$$
\begin{aligned}
\Phi_{\star}^{(k)}(\sigma) & =\frac{1}{2 i \pi} \int_{\Gamma}\left(\lambda-r_{\star}(\lambda, \sigma)\right) \phi^{(k)}(\lambda) d \lambda \\
& =\frac{k !}{2 i \pi} \int_{\Gamma}(\lambda-\sigma)^{\star(-k-1)} \phi(\lambda) d \lambda .
\end{aligned}
$$

If $\sigma=|\xi|^{q}+\sigma_{<q}$, with $\sigma_{<q}$ of order smaller than $q$, then the $\star$-resolvent reads

$$
r_{\star}\left(\lambda,|\xi|^{q}+\sigma_{<q}\right)=r_{\star}\left(\lambda,|\xi|^{q}\right)+\sum_{n=1}^{\infty} r_{\star n}\left(\lambda,|\xi|^{q}\right)\left(\sigma_{<q}\right)^{\otimes n},
$$


where, for symbols $\tau_{1}, \ldots, \tau_{n}$ in $\mathcal{F} \mathcal{S}_{\mathrm{cl}}(U)$, we have set

$$
\begin{aligned}
r_{\star n}\left(\lambda,|\xi|^{q}\right)\left(\tau_{1} \otimes \cdots \otimes \tau_{n}\right) \\
=\sum_{|k|=0}^{\infty} \frac{\left(k_{1}+\cdots+k_{n}+n-1\right) !}{k !\left(k_{1}+1\right)\left(k_{1}+k_{2}+1\right) \cdots\left(k_{1}+\cdots+k_{n-1}+n-1\right)} \\
\quad \cdot \operatorname{ad}_{|\xi|^{q}}^{\left(k_{1}\right)}\left(\tau_{1}\right) \star \operatorname{ad}_{|\xi|^{q}}^{\left(k_{2}\right)}\left(\tau_{2}\right) \star \cdots \star \operatorname{ad}_{|\xi|^{q}}^{\left(k_{n}\right)}\left(\tau_{n}\right)\left(\lambda-|\xi|^{q}\right)^{-|k|-n-1}
\end{aligned}
$$

with $|k|=k_{1}+\cdots+k_{n}$ and $k !=k_{1} ! \cdots k_{n}$ !

Second quantized functionals are defined on tensor products of symbols in terms of Cauchy integrals, by analogy with ordinary functionals on symbols (see (5.1)), but using quantized resolvents, $r_{\star n}$, instead of the ordinary resolvent, $r_{\star}$.

To an analytic function $\phi(z)$ and an admissible symbol $\sigma$, we assign a map called the second quantization of $\Phi(x)$, defined on $\left(\mathcal{F} \mathcal{S}_{\mathrm{cl}}(U)\right)^{\otimes n}$ by

$$
\begin{aligned}
\Phi_{\star n}(\sigma):\left(\mathcal{F S}_{\mathrm{cl}}(U)\right)^{\otimes n} & \rightarrow \mathcal{F} \mathcal{S}_{\mathrm{cl}}(U) \\
\tau_{1} \otimes \cdots \otimes \tau_{n} & \mapsto \frac{1}{2 i \pi} \int_{\Gamma} r_{\star n}(\lambda, \sigma)\left(\tau_{1} \otimes \cdots \otimes \tau_{n}\right) \phi(\lambda) d \lambda .
\end{aligned}
$$

One easily derives the following noncommutative Taylor-type formula from (5.4):

$$
\begin{aligned}
& \Phi_{\star n}(\sigma)\left(\tau_{1} \otimes \cdots \otimes \tau_{n}\right) \\
& \quad=\sum_{|k|=0}^{\infty} \frac{\operatorname{ad}_{\sigma}^{\star k_{1}}\left(\tau_{1}\right) \star \cdots \star \mathrm{ad}_{\sigma}^{\star k_{n}}\left(\tau_{n}\right)}{k !\left(k_{1}+1\right)\left(k_{1}+k_{2}+2\right) \cdots\left(k_{1}+\cdots+k_{n}+n\right)} \Phi^{(|k|+n)}(\sigma) .
\end{aligned}
$$

Applying (5.3) to $\sigma=|\xi|^{2}+\sigma_{<2}$, where $\sigma_{<2}$ has order smaller than two, we have

$$
\Phi_{\star}(\sigma)=\Phi_{\star}\left(|\xi|^{q}\right)+\sum_{p=1}^{\infty} \Phi_{\star p}\left(|\xi|^{q}\right)\left(\sigma_{<2}^{\otimes p}\right) .
$$

Applying this with $\phi=\log$ yields

$$
\begin{aligned}
\log _{\star}(\sigma) & -\log _{\star}\left(|\xi|^{2}\right) \\
= & \sum_{p=1}^{\infty} \sum_{|k|=0}^{\infty}(-1)^{|k|+p-1}(|k|+p-1) \\
& \cdot \frac{\operatorname{ad}_{|\xi|^{2}}^{\star k_{1}}\left(\sigma_{<2}\right) \star \operatorname{ad}_{|\xi|^{2}}^{\star k_{2}}\left(\sigma_{<2}\right) \cdots \star \operatorname{ad}_{|\xi|^{2}}^{\star k k_{p}}\left(\sigma_{<2}\right)}{k !\left(k_{1}+1\right)\left(k_{1}+k_{2}+2\right) \cdots\left(k_{1}+\cdots+k_{p}+p\right)}|\xi|^{-2(|k|+p)}
\end{aligned}
$$

Finally, implementing the noncommutative residue leads to the following formula for the logarithmic residue density. 
THEOREM 5.1. The logarithmic residue density of a generalized Laplacian $Q$ on $E$ is a finite sum of residues of classical symbols:

$$
\begin{aligned}
& \operatorname{res}_{x}(\log (Q))=\sum_{p=1}^{n} \sum_{|k|=0}^{n-p}(-1)^{|k|+p-1}(|k|+p-1) ! \\
& \quad \times \frac{\operatorname{res}_{x}\left(\left(L_{x}+\Delta_{x}\right)^{k_{1}}\left(\sigma_{<2}(Q)\right) \cdots\left(L_{x}+\Delta_{x}\right)^{k_{p}}\left(\sigma_{<2}(Q)\right)|\xi|^{-2(|k|+p)}\right)}{k !\left(k_{1}+1\right)\left(k_{1}+k_{2}+2\right) \cdots\left(k_{1}+\cdots+k_{p}+p\right)},
\end{aligned}
$$

where $k !:=k_{1} ! \cdots k_{p}$ ! and $|k|=k_{1}+\cdots+k_{p}$. Here we have set

$$
\sigma_{<2}(Q)(x, \xi):=\sigma(Q)(x, \xi)-|\xi|^{2}
$$

and, as before, $L_{x}:=-2 i \sum_{a=1}^{n} \xi_{a} \partial_{x_{a}}$ and $\Delta_{x}:=-\sum_{a=1}^{n} \partial_{x_{a}}^{2}$.

PROOF. By (5.7), combined with Example 4.3, we have

$$
\begin{aligned}
& \sigma(\log (Q))(x, \xi) \sim \sum_{p=1}^{\infty} \sum_{|k|=0}^{\infty}(-1)^{|k|+p-1}(|k|+p-1) ! \cdot \\
& \frac{\left(L_{x}+\Delta_{x}\right)^{k_{1}}\left(\sigma_{<2}(Q)\right) \cdots\left(L_{x}+\Delta_{x}\right)^{k_{p}}\left(\sigma_{<2}(Q)\right)|\xi|^{-2(|k|+p)}}{k !\left(k_{1}+1\right)\left(k_{1}+k_{2}+2\right) \cdots\left(k_{1}+\cdots+k_{p}+p\right)},
\end{aligned}
$$

which is a formal power series of symbols $\sigma_{k}$ of decreasing order $-(|k|+p)$. Since the noncommutative residue vanishes on symbols of order smaller than $-n$, we have $|k|+p \leq n$ which implies that only terms with $p \leq n$ and $|k| \leq n-p$ survive after applying the residue.

\section{The index as a logarithmic (super)residue}

We recall results from [9, 12] (see also [10]). Let $Q \in C \ell(M, E)$ be an admissible (and hence invertible, see Appendix A) classical pseudodifferential operator of positive order $q$.

For any differential operator $A \in C \ell(M, E)$, the noncommutative residue density

$$
\operatorname{res}_{x}(A \log Q) d x:=-\frac{1}{(2 \pi)^{n}}\left(\int_{|\xi|=1} \operatorname{tr}\left(\sigma_{-n}(A \log Q)(x, \xi)\right) d_{S} \xi\right) d x
$$

is a globally defined $n$-form on $M$ (see [7] for the case $A=I$, [9] for the general case), which integrates over $M$ to the noncommutative residue

$$
\operatorname{res}(A \log Q):=-\frac{1}{(2 \pi)^{n}} \int_{M}\left(\int_{|\xi|=1} \operatorname{tr}\left(\sigma_{-n}(A \log Q)(x, \xi)\right) d_{S} \xi\right) d x .
$$

Here tr corresponds to the fibrewise trace on $\operatorname{End}(E)$.

The residue, furthermore, relates to the $Q$-weighted trace $\operatorname{Tr}^{Q}(A)$ of $A$ by (see [12] when $A=I$ and [9] for the general case)

$$
\operatorname{Tr}^{Q}(A):=\mathrm{fp}_{z=0} \operatorname{Tr}\left(A Q^{-z}\right)=-\frac{1}{q} \operatorname{res}(A \log Q),
$$


where $\mathrm{fp}_{z=0}$ stands for the finite part at $z=0$ and $\operatorname{Tr}$ for the trace on trace-class operators. Here, $d_{S} \xi$ is the volume form on the unit sphere induced by the canonical measure on $\mathbb{R}^{n}$, where $\sigma_{-n}$ stands for the positively homogeneous component of degree $-n$ of a $\log$-polyhomogeneous symbol $\sigma$.

REMARK 6.1. One checks that

$$
\operatorname{res}(A \log (Q+R))=\operatorname{res}(A \log Q)
$$

for any smoothing operator $R$.

EXAMPLE 6.2. Setting $A=I$ in formula (6.3) yields

$$
\zeta_{Q}(0)=-\frac{1}{q} \operatorname{res}(\log Q)
$$

where $\zeta_{Q}(z)$ is the zeta function associated to $Q$. This corresponds to the logarithm,

$$
\operatorname{res}(\log Q)=\log \operatorname{det}_{\text {res }}(Q),
$$

of Scott's residue determinant [12].

Let $E=E^{+} \oplus E^{-}$be any $\mathbb{Z}_{2}$ graded vector bundle over $M$ and let

$$
D^{+}: C \ell\left(M, E_{+}\right) \rightarrow C \ell\left(M, E_{-}\right)
$$

be an elliptic operator in $C \ell\left(M, E_{+}^{*} \otimes E_{-}\right)$. Its (formal) adjoint,

$$
D^{-}:=\left(D^{+}\right)^{*}: C \ell\left(M, E^{-}\right) \rightarrow C \ell\left(M, E^{+}\right),
$$

is an elliptic operator in $C \ell\left(M,\left(E^{-}\right)^{*} \otimes E_{+}\right)$. The operator, $\Delta=\Delta^{+} \oplus \Delta^{-}$, where we define $\Delta^{+}:=D^{-} D^{+}, \Delta^{-}:=D^{+} D^{-}$, is a nonnegative (formally) self-adjoint elliptic operator.

The following theorem, which combines formulas due to McKean and Singer [5] and Seeley [13], expresses the index of $D^{+}$,

$$
\operatorname{ind}\left(D^{+}\right):=\operatorname{dim}\left(\operatorname{Ker}\left(D^{+}\right)\right)-\operatorname{dim}\left(\operatorname{Ker}\left(D^{-}\right)\right) \text {, }
$$

in terms of the superweighted trace of the identity. Let $\pi_{\Delta}$ denote the orthogonal projection onto the kernel of $\Delta$, which is finite-dimensional as $M$ is compact.

THEOREM 6.3. The superresidue

$$
\operatorname{sres}(\log (\Delta)):=-\frac{1}{(2 \pi)^{n}}\left(\int_{|\xi|=1} \operatorname{str}\left(\sigma_{-n, 0}(\log (\Delta))(x, \xi)\right) d_{S} \xi\right) d x
$$

is a globally defined $n$-form and we have

$$
\operatorname{ind}\left(D^{+}\right)=\operatorname{str}^{\Delta+\pi_{\Delta}}(I)=-\frac{1}{2 \operatorname{ord}(D)} \operatorname{sres}\left(\log \left(\Delta+\pi_{\Delta}\right)\right),
$$

where $\pi_{\Delta}$ is the orthogonal projection onto the kernel of $\Delta$ and $\operatorname{ord}(D)$ is the order of $D$. Here str denotes the supertrace on the graded fibres of $E$. 
REMARK 6.4. In view of Remark 6.1, one can drop the explicit mention of $\pi_{\Delta}$ and write sres $(\log \Delta)$, since the projection $\pi_{\Delta}$ is smoothing and the residue is invariant under translation by a smoothing operator.

PROOF. We first observe a property of the spectrum of $\Delta$ :

$$
\operatorname{Spec}\left(\Delta^{+}\right)-\{0\}=\operatorname{Spec}\left(\Delta^{-}\right)-\{0\} .
$$

Indeed,

$$
\Delta^{+} u_{+}=\lambda^{+} u_{+} \Longrightarrow \Delta_{-}\left(D^{+} u_{+}\right)=\lambda^{+} D^{+} u_{+} \quad \forall u_{+} \in C^{\infty}\left(M, E_{+}\right)
$$

so that an eigenvalue $\lambda^{+}$of $\Delta^{+}$, with eigenvector $u_{+}$, is an eigenvalue of $\Delta^{-}$, with eigenvector $D^{+} u_{+}$, provided that the latter does not vanish. The converse is proved similarly.

Let us denote by $\left\{\lambda_{n}^{+}: n \in \mathbb{N}\right\}$, the set of discrete eigenvalues of $\Delta^{+}$and, similarly, by $\left\{\lambda_{n}^{-}: n \in \mathbb{N}\right\}$, the set of discrete eigenvalues of $\Delta^{-}$. For any complex number $z$,

$$
\begin{aligned}
\operatorname{str}\left(\left(\Delta+\pi_{\Delta}\right)^{-z}\right) & =\sum_{n \in \mathbb{N}}\left(\lambda_{n}^{+}+\delta_{\lambda_{n}^{+}}\right)^{-z}-\sum_{n \in \mathbb{N}}\left(\lambda_{n}^{-}+\delta_{\lambda_{n}^{-}}\right)^{-z} \\
& =\sum_{\lambda_{n}^{+} \neq 0}\left(\lambda_{n}^{+}\right)^{-z}-\sum_{\lambda_{n}^{-} \neq 0}\left(\lambda_{n}^{-}\right)^{-z}+\operatorname{dim} \operatorname{Ker} \Delta^{+}-\operatorname{dim} \operatorname{Ker} \Delta^{-} \\
& =\operatorname{ind}\left(D^{+}\right) .
\end{aligned}
$$

Taking the finite part at $z=0$, therefore, yields

$$
\operatorname{ind}\left(D^{+}\right)=\operatorname{str}^{\Delta+\pi_{\Delta}}(I)=-\frac{1}{2} \operatorname{sres}(\log (\Delta)) .
$$

This concludes the proof.

EXAMPLE 6.5. With the notation introduced at the beginning of the paper, for a Dirac operator,

$$
D_{W}^{+}: C^{\infty}\left(M, S^{+} \otimes W\right) \rightarrow C^{\infty}\left(M, S^{-} \otimes W\right),
$$

on the $\mathbb{Z}_{2}$-graded spinor bundle $S=S^{+} \oplus S^{-}$over an even-dimensional spin manifold $M$,

$$
\operatorname{ind}\left(D_{W}^{+}\right)=-\frac{1}{2} \operatorname{sres}\left(\log \left(D_{W}^{2}\right)\right)=-\frac{1}{2} \int_{M} \operatorname{sres}_{x}\left(\log \left(D_{W}^{2}\right)\right) d x .
$$

The remainder of this paper deals with the computation of the logarithmic density of the square, $D^{2}$, of the Dirac operator $D$ acting on spinors.

\section{A formula for the index via the Lichnerowicz formula}

We first recall the Lichnerowicz formula (see, for example, [1, Theorem 3.52]) or, equivalently, the general Bochner identity (see [4, Theorem 8.2]), which relates the 
square $D_{W}^{2}$ of the twisted Dirac operator $D_{W}$ to the Laplace-Beltrami operator

$$
\begin{aligned}
\Delta^{E} & =-\operatorname{tr}\left(\nabla^{T^{*} M \otimes E} \nabla^{E}\right) \\
& =-\sum_{i=1}^{n}\left(\nabla^{T^{*} M \otimes E} \nabla^{E}\right)_{e_{i}, e_{i}} \\
& =-\sum_{i=1}^{n}\left(\nabla_{e_{i}}^{E} \nabla_{e_{i}}^{E}-\nabla_{\nabla_{e_{i}}^{E} e_{i}}^{E}\right)
\end{aligned}
$$

associated with the superconnection $\nabla^{E}$ on $E$. Here, $\nabla^{T^{*} M \otimes E}$ is the connection induced on the tensor product bundle, $T^{*} M \otimes E$, by the Levi-Civita connection on $M$ and the connection $\nabla^{E}$ on $E$. In addition, $\left\{e_{i}: i=1, \ldots, n\right\}$ is a local orthonormal tangent frame.

PROPOSITION 7.1. We have

$$
D_{W}^{2}=\Delta^{E}+R^{E}=\Delta^{E}+R^{W}+\frac{r_{M}}{4},
$$

where $r_{M}$ stands for the scalar curvature on $M$ and

$$
R^{E}:=\sum_{i<j} c\left(e_{i}\right) c\left(e_{j}\right)\left(\nabla^{E}\right)_{e_{i}, e_{j}}^{2}, \quad R^{W}:=\sum_{i<j} c\left(e_{i}\right) c\left(e_{j}\right)\left(\nabla^{W}\right)_{e_{i}, e_{j}}^{2} .
$$

In particular, for a flat auxiliary bundle,

$$
D_{W}^{2}=\Delta_{M}+\frac{r_{M}}{4}
$$

where $\Delta_{M}$ is the Laplace-Beltrami operator on the Riemannian manifold $M$.

PROOF. We choose a local orthonormal tangent frame $\left\{e_{i}: i=1, \ldots, n\right\}$ at the point $x \in M$ such that $\left(\nabla_{e_{i}}^{E}\right)_{x}=0$ for all $i \in\{1, \ldots, n\}$. Since $D_{W}=\sum_{i=1}^{n} c\left(e_{i}\right) \nabla_{e_{i}}^{E}$ at $x$,

$$
\begin{aligned}
D_{W}^{2} & =\sum_{i, j=1}^{n} c\left(e_{i}\right) \nabla_{e_{i}}^{E} c\left(e_{j}\right) \nabla_{e_{j}}^{E} \\
& =\sum_{i, j=1}^{n} c\left(e_{i}\right) c\left(e_{j}\right)\left[\left(\nabla^{E}\right)_{e_{i}, e_{j}}^{2}+\nabla_{\nabla_{e_{i}} e_{j}}^{E}\right] \\
& =-\sum_{i=1}^{n}\left(\nabla^{E}\right)_{e_{i}, e_{i}}^{2}+\sum_{i<j} c\left(e_{i}\right) c\left(e_{j}\right)\left[\left(\nabla^{E}\right)_{e_{i}, e_{j}}^{2}-\left(\nabla^{E}\right)_{e_{j}, e_{i}}^{2}\right] \\
& =\Delta^{E}+\sum_{i<j} c\left(e_{i}\right) c\left(e_{j}\right)\left(\nabla^{E}\right)_{e_{i}, e_{j}}^{2} \\
& =\Delta^{E}+R^{E} .
\end{aligned}
$$

The curvature term, $\left(\nabla^{E}\right)^{2} \in \Omega^{2}(M, \operatorname{End}(E))$, decomposes into

$$
\left(\nabla^{E}\right)^{2}=\left(\nabla^{S}\right)^{2} \otimes 1+1 \otimes\left(\nabla^{W}\right)^{2}
$$


so that

$$
R^{E}=\sum_{i<j} c\left(e_{i}\right) c\left(e_{j}\right)\left(\nabla^{S}\right)_{e_{i}, e_{j}}^{2}+R^{W} .
$$

A careful computation (see, for example, the proof of [1, Theorem 3.52]) shows that

$$
\sum_{i<j} c\left(e_{i}\right) c\left(e_{j}\right)\left(\nabla^{S}\right)_{e_{i}, e_{j}}^{2}=\frac{r_{M}}{4} .
$$

This concludes the proof.

Combining the Lichnerowicz formula with the Campbell-Hausdorff formula yields a formula for the index.

THEOREM 7.2. In even dimensions, $n=2 p$,

$$
\begin{aligned}
\operatorname{ind}\left(D_{W}^{+}\right) & =-\frac{1}{2} \operatorname{sres}\left(\log \left(D_{W}^{2}\right)\right) \\
& =-\frac{1}{2} \operatorname{sres}\left(\log \left(\Delta^{E}\right)\right)+\sum_{k=1}^{n-1} \frac{(-1)^{k}}{2 k} \operatorname{sres}\left(\left[\left(\Delta^{E}\right)^{-1} R^{E}\right]^{k}\right) .
\end{aligned}
$$

Inside the residue expansion we use the shorthand $\left(\Delta^{E}\right)^{-1}$ for $\left(\Delta^{E}+\pi_{\Delta}\right)^{-1}$, since the residue is insensitive to the smoothing operator $\pi_{\Delta}$.

PROOF. By equation (7.2),

$$
\begin{aligned}
D_{W}^{2}+\pi_{D_{W}^{2}} & =\Delta^{E}+\pi_{\Delta_{E}}+R^{E}+\pi_{D_{W}^{2}}-\pi_{\Delta^{E}} \\
& =\left(\Delta^{E}+\pi_{\Delta^{E}}\right)\left(1+\left(\Delta^{E}+\pi_{\Delta^{E}}\right)^{-1}\left(R^{E}+\pi_{D^{2}}-\pi_{\Delta^{E}}\right)\right),
\end{aligned}
$$

so that by (4.4), we obtain

$$
\begin{aligned}
\operatorname{sres}\left(\log \left(D_{W}^{2}\right)\right) & =\operatorname{sres}\left(\log \left(\Delta^{E}\right)\right)+\operatorname{sres}\left(\log \left(1+\left(\Delta^{E}\right)^{-1}\left(R^{E}\right)\right)\right) \\
& =\operatorname{sres}\left(\log \left(\Delta^{E}\right)\right)+\sum_{k=1}^{\infty} \frac{(-1)^{k+1}}{k} \operatorname{sres}\left(\left[\left(\Delta^{E}\right)^{-1}\left(R^{E}\right)\right]^{k}\right) \\
& =\operatorname{sres}\left(\log \left(\Delta^{E}\right)\right)+\sum_{k=1}^{\infty} \frac{(-1)^{k+1}}{k} \operatorname{sres}\left(\left[\left(\Delta^{E}\right)^{-1} \mathbb{R}^{E}\right]^{k}\right) .
\end{aligned}
$$

Here, we have used the fact that the noncommutative residue vanishes on smoothing operators. Also, for an operator $B \in C \ell(M, E)$ with negative order,

$$
\operatorname{sres}(\log (1+B))=\sum_{k=1}^{\infty} \frac{(-1)^{k+1}}{k} \operatorname{sres}\left(B^{k}\right),
$$

which is actually a finite sum since the residue vanishes for operators of order smaller than minus the dimension of the underlying manifold. Since $B=\left(\Delta^{E}+\pi_{\Delta}\right)^{-1} R^{E}$ has order -2 , the sum stops at $p=n / 2$.

\section{The Atiyah-Singer index theorem for a twisted Dirac operator on a flat space}

We derive the Atiyah-Singer index formula for a twisted Dirac operator on a flat space from (7.4). We use the notation introduced at the beginning of the paper. 
We denote the components of the connection $\nabla^{E}$ in a given local trivialization of $E$ by $\partial_{i}+A_{i}$. We take local coordinates $x_{i}$ on $M$ such that the metric Christoffel symbols vanish. By the Lichnerowicz formula,

$$
D_{W}^{2}=\Delta^{E}+R^{E}=\sum_{i}\left(\partial_{i}+A_{i}\right)^{2}+\sum_{i<j} \gamma_{i} \gamma_{j} F_{i j},
$$

where we have set

$$
F_{i j}=\partial_{i} A_{j}-\partial_{j} A_{i}+\left[A_{i}, A_{j}\right]
$$

to be the 2-form components of the curvature, $\left(\nabla^{E}\right)^{2}$.

THEOREM 8.1. If the Riemann metric on $M$ is flat, then

$$
\operatorname{ind}\left(D_{W}^{+}\right)=\int_{M} \operatorname{tr}\left(e^{i(F / 2 \pi)}\right)
$$

ProOf. As before, $n=2 p$ denotes the dimension of $M$. By Theorem 7.2,

$$
\text { sres } \log \left(D_{W}{ }^{2}\right)=\operatorname{sres}\left(\log \Delta^{E}\right)+\sum_{k=1}^{n-1} \frac{(-1)^{k+1}}{k} \operatorname{sres}\left(\left[\left(\Delta^{E}\right)^{-1} R^{E}\right]^{k}\right) .
$$

The first term on the right-hand side vanishes. Indeed, at a given point $x \in M$ and, for fixed $\xi \in T_{x}^{*} M$, the $-n$th homogeneous component of the symbol, $\sigma\left(\log \Delta^{E}\right)(x, \xi)$, of $\log \Delta^{E}$ is an endomorphism of the fibre $W_{x}$ of the auxiliary vector bundle $W$. By (2.1), the fibrewise supertrace therefore vanishes on the $-n$th homogeneous component of the symbol and, hence, so does the residue density $\operatorname{sres}_{x}\left(\log \Delta^{E}\right) d x$. It follows that $\operatorname{sres}\left(\log \Delta^{E}\right)=0$.

We now investigate the second term on the right-hand side. On the one hand, all the expressions

$$
\text { sres } \log \left(\left[\left(\Delta^{E}\right)^{-1} R^{E}\right]^{k}\right)
$$

inside the sum vanish for $k>p$. Whenever the operators $\left[\left(\Delta^{E}\right)^{-1} R^{E}\right]^{k}$ are of order smaller than $-n$, their residues vanish.

On the other hand, the expressions inside the sum also vanish for $k<p$. Indeed, at a point $x \in M$ and, for fixed $\xi \in T_{x}^{*} M$, the symbols in the variables $(x, \xi)$ inside the residues are of the form $M \gamma_{i_{1}} \gamma_{i_{2}} \cdots \gamma_{i_{k}}$ for some matrix $M \in \operatorname{End}\left(W_{x}\right)$ and proper subsets $\left\{i_{1}, \ldots, i_{k}\right\}$ of $\{1, \ldots, n\}$. Their supertraces, which arise inside the superresidue, therefore vanish by (2.1).

The remaining term in the sum at $k=p$ corresponds to the residue of an operator of order $-n$. It only involves the leading symbol $\sigma_{L}\left(\Delta^{E}\right)=|\xi|^{2}$ of $\Delta^{E}$. Thus, we obtain

$$
\begin{aligned}
\operatorname{sres} \log \left(D_{W}^{2}\right) & =-\frac{(-1)^{p}}{p} \operatorname{sres}\left(\left[\left(\Delta^{E}+\pi_{\Delta^{E}}\right)^{-1} R^{E}\right]^{p}\right) \\
& =-\frac{(-1)^{p}}{p} \operatorname{sres}\left(|\xi|^{-n}\left(\operatorname{tr}\left(R^{E}\right)\right)^{p}\right) \\
& =-\frac{(-1)^{p}}{p} \int_{M} \operatorname{sres}_{x}\left(|\xi|^{-n}\left(\sum_{i<j} \gamma_{i} \gamma_{j} F_{i j}\right)^{p}\right) d x \quad \text { by (7.3) }
\end{aligned}
$$




$$
\begin{aligned}
& =-\frac{(-1)^{p} 2^{p}\left(S^{n-1}\right)}{(2 \pi)^{n} p} \int_{M} \operatorname{str}\left(\sum_{i, j} \sigma_{i j} F_{i j}\right)^{p} d x \quad \text { by (2.2) and (1.1) } \\
& =-2 \frac{i^{p}}{(4 \pi)^{p} p !} \sum_{\tau \in \Sigma_{n}}(-1)^{|\tau|} \int_{M} F_{\tau(1) \tau(2)} \cdots F_{\tau(n-1) \tau(n)} d x
\end{aligned}
$$

by (2.3) and (8.1)

$$
\begin{aligned}
& =-2 \frac{i^{p}}{(2 \pi)^{p} p !} \int_{M} \operatorname{tr}\left(F^{\wedge p}\right) d x \\
& =-2 \int_{M} \operatorname{tr}\left(e^{i F / 2 \pi}\right) .
\end{aligned}
$$

In the above calculation, we have used the identity

$$
\operatorname{vol}\left(S^{n-1}\right)=\frac{2 \pi^{n / 2}}{\Gamma(n / 2)}=\frac{2 \pi^{p}}{(p-1) !} .
$$

This concludes the proof.

\section{The curvature tensor in normal coordinates}

We recall a few properties of the curvature in a normal local coordinate system, that is, a coordinate system defined by the exponential map at a point, so that rays emanating from the origin in the tangent space at this point are mapped to geodesics on the manifold emanating from this point. Let us recall that, in Riemannian normal coordinates (see, for example, [1, Proposition 1.28]),

$$
g_{i j}=\partial_{i j}-\frac{1}{3} R_{i k j l} x^{l} x^{k}+\sum_{|\alpha| \geq 3} \partial_{\alpha} g_{i j} \frac{x^{\alpha}}{\alpha !} .
$$

LEMMA 9.1. We have

$$
\left(R_{i a j k}+R_{i k j a}\right) \sigma_{k j}=\frac{3}{2} R_{i a j k} \sigma_{k j},
$$

where $\sigma_{i j}$ was defined in (2.2).

PROOF. Using the first Bianchi identity,

$$
R_{[i j k] l}=0 \text {, }
$$

we write $R_{i j k a}=-R_{k i j a}-R_{j k i a}$ which, combined with the antisymmetry of $\sigma_{i j}$ in $i$ and $j$, and the (anti)symmetry properties of the curvature tensor

$$
R_{i j k l}=-R_{j i k l}=-R_{i j l k}=R_{k l i j}
$$

yields

$$
\begin{aligned}
\left(R_{i a j k}+R_{i k j a}\right) \sigma_{k j} & =R_{i a j k} \sigma_{k j}+R_{i j k a} \sigma_{j k} \\
& =\left(R_{i a j k}+R_{k i j a}+R_{j k i a}\right) \sigma_{k j} \\
& =\left(2 R_{i a j k}-R_{i k j a}\right) \sigma_{k j} .
\end{aligned}
$$


Consequently,

$$
\begin{aligned}
\left(R_{i a j k}+R_{i k j a}\right) \sigma_{k j} & =\frac{1}{2}\left[\left(R_{i a j k}+R_{i k j a}\right) \sigma_{k j}+\left(2 R_{i a j k}-R_{i k j a}\right) \sigma_{k j}\right] \\
& =\frac{3}{2} R_{i a j k} \sigma_{k j} .
\end{aligned}
$$

This concludes the proof.

PROPOSITION 9.2. At the centre of a normal coordinate system,

$$
\partial_{a} \Gamma_{i j}^{k} \sigma_{k j}=\frac{1}{2} R_{j k i a} \sigma_{k j}
$$

so that $\partial_{i} \Gamma_{i j}^{k} \sigma_{k j}=0$.

PROOF. By (9.1), the Christoffel symbols $\Gamma_{i j}^{k}=\frac{1}{2} g^{k l}\left(\partial_{j} g_{i l}+\partial_{i} g_{j l}-\partial_{l} g_{i j}\right)$ vanish at the centre of the normal coordinate system, where we have

$$
\partial_{a} \Gamma_{i j}^{k}=\frac{1}{3}\left(R_{i a j k}+R_{j a i k}\right) .
$$

Indeed, differentiating (9.1) twice yields

$$
\begin{aligned}
\partial_{a} \Gamma_{i j}^{k} & =\frac{1}{2} \delta^{k l}\left(\partial_{a} \partial_{j} g_{i l}+\partial_{a} \partial_{i} g_{j l}-\partial_{a} \partial_{l} g_{i j}\right) \\
& =-\frac{1}{6}\left(R_{i a k j}+R_{i j k a}+R_{j a k i}+R_{j i k a}-R_{i a j k}-R_{i k j a}\right) \\
& =\frac{1}{3}\left(R_{i a j k}+R_{j a i k}\right) .
\end{aligned}
$$

It follows from Lemma 9.1 that $\partial_{a} \Gamma_{i j}^{k} \sigma_{k j}=\frac{1}{2} R_{i a j k} \sigma_{k j}$, and hence, in particular, that

$$
\partial_{i} \Gamma_{i j}^{k} \sigma_{k j}=\frac{1}{2} R_{i a j k} \sigma_{k j}=0
$$

at the centre of the normal coordinate system.

The following result is useful when computing the index.

PROPOSITION 9.3. In four dimensions,

$$
\operatorname{sres}_{x}\left(|\xi|^{-4} \partial_{x_{a}} \Gamma_{i j}^{k} \partial_{x_{a}} \Gamma_{i m}^{n} \sigma_{k j} \sigma_{n m}\right) d x=\frac{1}{32 \pi^{2}} \operatorname{tr}(R \wedge R)
$$

and

$$
\operatorname{sres}_{x}\left(\frac{\xi_{a} \xi_{b}}{|\xi|^{6}} \partial_{x_{a}} \Gamma_{i j}^{k} \partial_{x_{b}} \Gamma_{i m}^{n}\right) d x=\frac{1}{4 \times 32 \pi^{2}} \operatorname{tr}(R \wedge R) .
$$

PROOF. The result in four dimensions is a consequence of the following formula in $n=2 p=4 q$ dimensions. At the centre of a normal coordinate system, we show that

$$
\begin{aligned}
& \operatorname{sres}_{x}\left(|\xi|^{-2 p} \partial_{x_{a_{1}}} \Gamma_{i_{1} j_{1}}^{k_{1}} \partial_{x_{a_{1}}} \Gamma_{i_{1} m_{1}}^{n_{1}} \cdots\right. \\
& \left.\quad \times \partial_{x_{a_{p}}} \Gamma_{i_{q} j_{q}}^{k_{q}} \partial_{x_{a_{q}}} \Gamma_{i_{q} m_{q}}^{n_{q}} \sigma_{k_{1} j_{1}} \sigma_{n_{1} m_{1}} \cdots \sigma_{k_{q} j_{q}} \sigma_{n_{q} m_{q}}\right) d x \\
& \quad=\frac{1}{\Gamma(p) 2^{3 p-1} \pi^{p}}(\operatorname{tr}(R \wedge R))^{q} .
\end{aligned}
$$


The proof follows by combining (9.2) with (6.1) and the formula for the volume of the unit sphere $S^{n-1}$ in $n$ dimensions given by (8.1):

$$
\begin{aligned}
& \operatorname{sres}_{x}\left(|\xi|^{-2 p} \partial_{x_{a_{1}}} \Gamma_{i_{1} j_{1}}^{k_{1}} \partial_{x_{a_{1}}} \Gamma_{i_{1} m_{1}}^{n_{1}} \cdots \partial_{x_{a_{q}}} \Gamma_{i_{q} j_{q}}^{k_{q}} \partial_{x_{a_{q}}} \Gamma_{i_{q} m_{q}}^{n_{q}} \sigma_{k_{1} j_{1}} \sigma_{n_{1} m_{1}} \cdots \sigma_{k_{q} j_{q}} \sigma_{n_{q} m_{q}}\right) \\
&=\frac{1}{2^{2 q}} \operatorname{sres}_{x}\left(|\xi|^{-2 p} R_{i_{1} a_{1} j_{1} k_{1}} R_{i_{1} a_{1} m_{1} n_{1}} \cdots R_{i_{q} a_{q} j_{q} k_{q}} R_{i_{q} a_{q} m_{q} n_{q}}\right. \\
&\left.\quad \times \sigma_{k_{1} j_{1}} \sigma_{n_{1} m_{1}} \cdots \sigma_{k_{q} j_{q}} \sigma_{n_{q} m_{q}}\right) \\
&=\frac{(-i)^{p}}{4 p} \sum_{\tau \in \Sigma_{n}}(-1)^{|\tau|} \operatorname{res}_{x}\left(|\xi|^{-2 p} R_{i_{1} a_{1} \tau(1) \tau(2)} \cdots R_{i_{q} a_{q} \tau(n-3) \tau(n-2)}\right. \\
&\left.\times R_{i_{q} a_{q} \tau(n-1) \tau(n)}\right) \\
&= \frac{1}{\Gamma(p) 2^{2 n-1} \pi^{p}} \sum_{\tau \in \Sigma_{n}}(-1)^{|\tau|} R_{i_{1} a_{1} \tau(1) \tau(2)} \cdots R_{i_{q} a_{q} \tau(n-3) \tau(n-2)} R_{a_{q} i_{q} \tau(n-1) \tau(n)}
\end{aligned}
$$

by (9.2), (2.1) and (2.3). On the other hand,

$$
\begin{aligned}
(\operatorname{tr}(R \wedge & R))^{q} \\
= & \frac{1}{2^{p}} \sum_{\tau \in \Sigma_{n}}(-1)^{|\tau|} R_{i_{1} a_{1} \tau(1) \tau(2)} R_{a_{1} i_{1} \tau(3) \tau(4)} \ldots R_{i_{q} a_{q} \tau(n-3) \tau(n-2)} \\
& \times R_{a_{q} i_{q} \tau(n-1) \tau(n)} d x
\end{aligned}
$$

which yields

$$
\begin{aligned}
& \operatorname{sres}_{x}\left(|\xi|^{-2 p} \partial_{x_{a_{1}}} \Gamma_{i_{1} j_{1}}^{k_{1}} \partial_{x_{a_{1}}} \Gamma_{i_{1} m_{1}}^{n_{1}} \cdots \partial_{x_{a_{q}}} \Gamma_{i_{q} j_{q}}^{k_{q}} \partial_{x_{a_{q}}} \Gamma_{i_{q} m_{q}}^{n_{q}} \sigma_{k_{1} j_{1}} \sigma_{n_{1} m_{1}} \cdots \sigma_{k_{q} j_{q}} \sigma_{n_{q} m_{q}}\right) d x \\
& =\frac{1}{\Gamma(p) 2^{3 p-1} \pi^{p}}(\operatorname{tr}(R \wedge R))^{q} .
\end{aligned}
$$

This proves the first part of the statement.

We prove the second part similarly. We first observe that, using the symmetries of the sphere,

$$
\begin{aligned}
\int_{|\xi|=1} \frac{\xi_{i} \xi_{j}}{|\xi|^{n+2}} d \xi & =\delta_{i-j} \int_{|\xi|=1} \frac{\xi_{i}^{2}}{|\xi|^{n+2}} d \xi \\
& =\frac{1}{n} \delta_{i-j} \int_{|\xi|=1} \frac{\sum_{i=1}^{n} \xi_{i}^{2}}{|\xi|^{n+2}} d \xi \\
& =\frac{\delta_{i-j}}{n} \frac{2 \pi^{n / 2}}{\Gamma\left(\frac{n}{2}\right)} .
\end{aligned}
$$

Thus, in four dimensions, we obtain

$$
\operatorname{sres}_{x}\left(\frac{\xi_{a} \xi_{b}}{|\xi|^{6}} \partial_{x_{a}} \Gamma_{i j}^{k} \partial_{x_{b}} \Gamma_{i m}^{n}\right) d x=\frac{1}{4 \times 32 \pi^{2}} \operatorname{tr}(R \wedge R) .
$$

This concludes the proof. 


\section{The Atiyah-Singer index formula in four dimensions}

The square of a Dirac operator $D$ acting on pure spinors is the prototype of a generalized Laplacian. In local coordinates, its symbol reads

$$
\sigma\left(D^{2}\right)=|\xi|^{2}+\Gamma_{i j}^{k} \sigma_{k j} \xi_{i}+\partial_{i} \Gamma_{i j}^{k} \sigma_{k j}+\Gamma_{i j}^{k} \Gamma_{i m}^{n} \sigma_{k j} \sigma_{n m}+s=|\xi|^{2}+\sigma_{<2}\left(D^{2}\right),
$$

where

$$
\sigma_{<2}\left(D^{2}\right):=\Gamma_{i j}^{k} \sigma_{k j} \xi_{i}+\partial_{i} \Gamma_{i j}^{k} \sigma_{k j}+\Gamma_{i j}^{k} \Gamma_{i m}^{n} \sigma_{k j} \sigma_{n m}+s,
$$

and $s$ denotes the scalar curvature.

We use (6.6) to compute the index of $D^{+}$,

$$
\operatorname{ind}\left(D^{+}\right)=-\frac{1}{2} \int_{M} \operatorname{sres}_{x}\left(\log D^{2}\right) d x,
$$

in terms of the logarithmic (super)residue density, which we explicitly derive in four dimensions.

Since the residue does not depend on the choice of local coordinates, we choose to derive the residue density in a normal coordinate system. We therefore need to compute

$$
\operatorname{sres}_{x}\left(\log D^{2}\right)=\operatorname{sres}_{x}\left(\log _{\star}\left(|\xi|^{2}+\sigma_{<2}\left(D^{2}\right)\right)\right)
$$

where $\log _{\star}$ is the logarithm on symbols. There are at least two methods to compute the logarithm of $|\xi|^{2}+\sigma_{<2}\left(D^{2}\right)$. These are the Campbell-Hausdorff formula (4.9) and a Taylor-type formula as in (5.8).

In the following, we let $\sigma_{<k}$ denote the part of the symbol $\sigma$ of order smaller than $k$. Using the first method, we obtain the following expansion in four dimensions:

$$
\begin{aligned}
\operatorname{sres}_{x}( & \left.\log \left(D^{2}\right)\right) \\
= & \operatorname{sres}_{x}\left(\log _{\star}\left(|\xi|^{-2} \star \sigma\left(D^{2}\right)\right)\right) \\
& +\frac{1}{2} \sum_{j=0}^{2} \frac{(-1)^{j}}{(j+1) !} \operatorname{sres}_{x}\left(\left(\operatorname { l o g } | \xi | ^ { 2 } \star \left(|\xi|^{-2} \star \sigma_{<2}\left(D^{2}\right)\right.\right.\right. \\
& \left.\left.\times(x, \xi))^{*(j+1)}\right)_{<-j-1}\right) \\
& +\frac{1}{12} \sum_{j=0}^{1} \frac{(-1)^{j}}{(j+1) !} \\
& \cdot \operatorname{sres}_{x}\left(\left(\operatorname { l o g } | \xi | ^ { 2 } \star \left(\operatorname { l o g } | \xi | ^ { 2 } \star \left(|\xi|^{-2} \star \sigma_{<2}\left(D^{2}\right)\right.\right.\right.\right. \\
& \left.\left.\left.\times(x, \xi))^{*(j+1)}\right)_{<-j-1}\right)_{<-j-2}\right) \\
& -\frac{1}{12} \operatorname{sres}_{x}\left(\left\{|\xi|^{-2} \star \sigma_{<2}\left(D^{2}\right),\left(\operatorname { l o g } | \xi | ^ { 2 } \star \left(|\xi|^{-2} \star \sigma_{<2}\right.\right.\right.\right. \\
& \left.\left.\left.\left.\times\left(D^{2}\right)(x, \xi)\right)\right)_{<-1}\right\}_{\star}\right) .
\end{aligned}
$$

As this requires the computation of various terms in the above sums, it is lengthier than the second method, which we shall adopt. 
Replacing the residue by a superresidue in (5.8) yields the following description of the logarithmic superresidue density of $D^{2}$, in which the sum over $p$ reduces to one term.

PROPOSITION 10.1. The logarithmic superresidue density of the squared Dirac operator is a finite sum of superresidues of classical symbols:

$$
\begin{aligned}
\operatorname{sres}_{x} & \left(\log \left(D^{2}\right)\right) \\
= & \sum_{|k|=n / 4, k_{i} \in\{1,2\}}^{n / 2} \frac{(-1)^{|k|+q-1}(|k|+q-1) !}{k !\left(k_{1}+1\right)\left(k_{1}+k_{2}+2\right) \cdots\left(k_{1}+\cdots+k_{p}+p\right)} \\
& \quad \times \operatorname{sres}_{x}\left(\left(L_{x}+\Delta_{x}\right)^{k_{1}}\left(\sigma_{<2}\left(D^{2}\right)\right) \cdots\left(L_{x}+\Delta_{x}\right)^{k_{q}}\left(\sigma_{<2}\left(D^{2}\right)\right)|\xi|^{-2(|k|+q)}\right)
\end{aligned}
$$

where, as before, we have set $q=n / 4$. It is of the form

$$
\operatorname{sres}_{x}\left(\log \left(D^{2}\right)\right)=\sum_{s+t=q} \alpha_{s, t} \operatorname{sres}_{x}\left(\left(L_{x}^{2} \sigma_{<2}\left(D^{2}\right)\right)^{s}\left(\Delta_{x} \sigma_{<2}\left(D^{2}\right)\right)^{t}|\xi|^{-2(3 s+2 t)}\right),
$$

with $\Delta_{x} \sigma_{<2}\left(D^{2}\right)$ and $L_{x}^{2} \sigma_{<2}\left(D^{2}\right)$ contributing, respectively, by

$$
\Delta_{x}\left(\Gamma_{i j}^{k} \Gamma_{l m}^{n}\right) \sigma_{j k} \sigma_{m n}=-\frac{1}{2} R_{j k i a} R_{n m i a} \sigma_{k j} \sigma_{n m}
$$

and

$$
L_{x}^{2}\left(\Gamma_{i j}^{k} \Gamma_{l m}^{n}\right) \sigma_{j k} \sigma_{m n}=-R_{j k i a} R_{m n i b} \sigma_{j k} \sigma_{m n} \xi_{a} \xi_{b}
$$

Proof. Applying (5.8) to $Q=D^{2}$ yields an expression which involves terms of the form

$$
\left(L_{x}+\Delta_{x}\right)^{k_{i}}\left(\sigma_{<2}\left(D^{2}\right)\right)
$$

each of which differentiates $\sigma_{<2}\left(D^{2}\right)$ at least $k_{i}$ times. We have $k_{i} \leq 2$. Indeed, since $\operatorname{sres}_{x}\left(\log _{\star}\left(D^{2}\right)\right)$ is proportional to a Pontryagin form, it only involves curvature terms, and so only first-order derivatives of the Christoffel symbols can arise. The product term, $\Gamma_{i j}^{k} \Gamma_{i m}^{n}$, can only involve partial differential operators of order at most two. Since the superresidue density $\operatorname{sres}_{x}\left(\log D^{2}\right) d x$ is proportional to a Pontryagin form, there is no contribution from the scalar curvature, so that terms $\sigma_{<2}\left(D^{2}\right)$ corresponding to zero powers $k_{i}$ make no contribution.

We now analyse the contribution of terms involving powers $k_{i}=1$, that is, expressions of the type $\left(L_{x}+\Delta_{x}\right) \sigma_{<2}\left(D^{2}\right)$. By $(10.1)$, the terms $\Delta_{x} \sigma_{<2}\left(D^{2}\right)$ can only contribute by

$$
\begin{aligned}
\Delta_{x}\left(\Gamma_{i j}^{k} \Gamma_{i m}^{n}\right) \sigma_{k j} \sigma_{n m} & =-2 \partial_{a} \Gamma_{i j}^{k} \partial_{a} \Gamma_{i m}^{n} \sigma_{k j} \sigma_{n m} \\
& =-\frac{1}{2} R_{j k i a} R_{n m i a} \sigma_{k j} \sigma_{n m} .
\end{aligned}
$$

Let us now see how the terms $L_{x} \sigma_{<2}\left(D^{2}\right)$ contribute. We have

$$
\begin{aligned}
L_{x} \sigma_{<2}\left(D^{2}\right)=- & 2 i\left(\partial_{a} \Gamma_{i j}^{k} \sigma_{k j} \xi_{i} \xi_{a}+\partial_{a} \partial_{i} \Gamma_{i j}^{k} \sigma_{k j} \xi_{a}\right. \\
& \left.+\left(\partial_{a} \Gamma_{i j}^{k} \Gamma_{l m}^{n}+\Gamma_{i j}^{k} \partial_{a} \Gamma_{i m}^{n}\right) \sigma_{k j} \sigma_{n m} \xi_{a}+\partial_{a} s \xi_{a}\right),
\end{aligned}
$$


which, at the centre of a normal coordinate system, reads

$$
L_{x} \sigma_{<2}\left(D^{2}\right)=-2 i\left(\partial_{a} \Gamma_{i j}^{k} \sigma_{k j} \xi_{i} \xi_{a}+\partial_{a} \partial_{i} \Gamma_{i j}^{k} \sigma_{k j} \xi_{a}+\partial_{a} s \xi_{a}\right)
$$

The only possible contribution can come from

$$
L_{x}\left(\Gamma_{i j}^{k} \sigma_{k j} \xi_{i}\right)=-2 i \partial_{a} \Gamma_{i j}^{k} \sigma_{k j} \xi_{i} \xi_{a}=-i R_{j k i a} \sigma_{k j} \xi_{i} \xi_{a}
$$

which vanishes by antisymmetry of $R$. There is therefore no contribution from terms of the type $L_{x} \sigma_{<2}\left(D^{2}\right)$.

When $k_{i}=2$ we obtain terms of the form

$$
\left(L_{x}+\Delta_{x}\right)^{2} \sigma_{<2}\left(D^{2}\right)=L_{x}^{2} \sigma_{<2}\left(D^{2}\right)+2 L_{x} \Delta_{x} \sigma_{<2}\left(D^{2}\right)+\Delta_{x}^{2} \sigma_{<2}\left(D^{2}\right),
$$

and only $L_{x}^{2} \sigma_{<2}\left(D^{2}\right)$ contributes. This introduces terms of the type

$$
\begin{aligned}
L_{x}^{2}\left(\Gamma_{i j}^{k} \Gamma_{i m}^{n} \sigma_{k j} \sigma_{n m}\right) & =-4 \partial_{a} \Gamma_{i j}^{k} \partial_{b} \Gamma_{i m}^{n} \sigma_{k j} \sigma_{n m} \xi_{a} \xi_{b} \\
& =-R_{j k i a} R_{m n i b} \sigma_{j k} \sigma_{m n} \xi_{a} \xi_{b}
\end{aligned}
$$

In summary, we only have contributions from $\Delta_{x} \sigma_{<2}\left(D^{2}\right)$ and $L_{x}^{2} \sigma_{<2}\left(D^{2}\right)$ via products

$$
\left(L_{x}^{2} \sigma_{<2}\left(D^{2}\right)\right)^{s}\left(\Delta_{x} \sigma_{<2}\left(D^{2}\right)\right)^{t} \quad \text { with } p=s+t \text { and }|k|=2 s+t .
$$

Since the residue picks the $-n$th power in $|\xi|$, we have $2 s-2(|k|+q)=-n$ which implies that $2 s+2 t=n / 2$ and, hence, $q=n / 4$. This is confirmed by counting the Clifford coefficients, since (2.1) implies that

$$
2 q=2 s+2 t=\frac{n}{2} .
$$

Our result now follows from the above computations, together with (10.5) and (10.7).

EXAMPLE 10.2. When $n=4$, in which case $q=1$, we have $s+t=1$ so that we need to consider two types of terms: $\Delta_{x} \sigma_{<2}\left(D^{2}\right)$ and $L_{x}^{2}\left(\sigma_{<2}\left(D^{2}\right)\right)$.

Proposition 10.1, combined with Proposition 9.3, yields

$$
\begin{aligned}
\operatorname{sres}_{x}( & \left.\log \left(D^{2}\right)\right) \\
= & -\frac{1}{2} \operatorname{sres}_{x}\left(\Delta_{x}\left(\sigma_{<2}\left(D^{2}\right)\right)|\xi|^{-4}\right) \quad\left(\text { when } s=0, t=1, k_{1}=1\right) \\
& +\frac{\operatorname{sres}_{x}\left(L_{x}^{2}\left(\sigma_{<2}\left(D^{2}\right)\right)|\xi|^{-6}\right)}{2 \times 3} \quad\left(\text { when } s=1, t=0, k_{1}=2\right) \\
= & \operatorname{sres}_{x}\left(|\xi|^{-4} \partial_{a} \Gamma_{i j}^{k} \partial_{a} \Gamma_{i m}^{n} \sigma_{k j} \sigma_{n m}\right) \quad \text { by }(10.5) \\
& -\frac{4}{3} \operatorname{sres}_{x}\left(|\xi|^{-6} \partial_{a} \Gamma_{i j}^{k} \partial_{b} \Gamma_{i m}^{n} \sigma_{k j} \sigma_{n m} \xi_{a} \xi_{b}\right) \quad \text { by (10.7) }
\end{aligned}
$$




$$
\begin{aligned}
& =\left(1-\frac{1}{3}\right) \operatorname{sres}_{x}\left(|\xi|^{-4} \partial_{a} \Gamma_{i j}^{k} \partial_{a} \Gamma_{i m}^{n} \sigma_{k j} \sigma_{n m}\right) \quad \text { by (9.4) } \\
& =\frac{1}{48 \pi^{2}} \operatorname{tr}(R \wedge R) \quad \text { by (9.3). }
\end{aligned}
$$

Once integrated over the manifold $M$, this yields the well-known formula

$$
\begin{aligned}
\operatorname{ind}(D) & =\int_{M} \hat{A} \Longrightarrow \\
\operatorname{sres}\left(\log \left(D^{2}\right)\right) & =-2 \int_{M} \hat{A}=\frac{1}{48 \pi^{2}} \operatorname{tr}(R \wedge R),
\end{aligned}
$$

since

$$
\hat{A}=1-\frac{1}{24(2 \pi)^{2}} \operatorname{tr}(R \wedge R)+\cdots \text {. }
$$

\section{Appendix A. Complex powers and logarithms}

An operator $A \in C \ell(M, E)$ has principal angle $\theta$ if, for every $(x, \xi) \in T^{*} M-\{0\}$, the leading symbol, $\left(\sigma_{A}(x, \xi)\right)^{L}$, has no eigenvalue on the ray $L_{\theta}=\left\{r e^{i \theta}: r \geq 0\right\}$; in that case $A$ is elliptic.

Definition A.1. We call an operator $A \in C \ell(M, E)$ admissible with spectral cut $\theta$ if $A$ has principal angle $\theta$, and the spectrum of $A$ does not meet $\mathrm{L}_{\theta}=\left\{r e^{i \theta}: r \geq 0\right\}$. In particular, such an operator is invertible and elliptic. Since the spectrum of $A$ does not meet $L_{\theta}, \theta$ is called an Agmon angle of $A$.

Let $A \in C \ell(M, E)$ be admissible with spectral cut $\theta$ and positive order $a$. For $\operatorname{Re}(z)<0$, the complex power $A_{\theta}^{z}$ of $A$, first introduced by Seeley [13], is defined by the Cauchy integral

$$
A_{\theta}^{z}=\frac{i}{2 \pi} \int_{\Gamma_{r, \theta}} \lambda_{\theta}^{z}(A-\lambda)^{-1} d \lambda,
$$

where $\lambda_{\theta}^{z}=|\lambda|^{z} e^{i z(\arg \lambda)}$ with $\theta \leq \arg \lambda<\theta+2 \pi$. In particular, for $z=0$, we have $A_{\theta}^{0}=I$.

Here,

$$
\Gamma_{r, \theta}=\Gamma_{r, \theta}^{1} \cup \Gamma_{r, \theta}^{2} \cup \Gamma_{r, \theta}^{3},
$$

where

$$
\begin{gathered}
\Gamma_{r, \theta}^{1}=\left\{\rho e^{i \theta}: \infty>\rho \geq r\right\}, \\
\Gamma_{r, \theta}^{2}=\left\{\rho e^{i(\theta-2 \pi)}: \infty>\rho \geq r\right\}, \\
\Gamma_{r, \theta}^{3}=\left\{r e^{i t}: \theta-2 \pi \leq t \leq \theta\right\}
\end{gathered}
$$

is a contour along the ray $L_{\theta}$ around the nonzero spectrum of $A$. Here, $r$ is any small positive real number such that $\Gamma_{r, \theta} \cap \operatorname{Sp}(A)=\emptyset$.

The definition of complex powers can be extended to the whole complex plane by setting $A_{\theta}^{z}:=A^{k} A_{\theta}^{z-k}$ for $k \in \mathbb{N}$ and $\operatorname{Re}(z)<k$. This definition is independent of 
the choice of $k$ in $\mathbb{N}$ and preserves the usual properties, that is, $A_{\theta}^{z_{1}} A_{\theta}^{z_{2}}=A_{\theta}^{z_{1}+z_{2}}$ and $A_{\theta}^{k}=A^{k}$, for $k \in \mathbb{Z}$.

The complex powers of an admissible operator, $A \in C \ell(M, E)$ with zero order and spectral cut $\theta$, give rise to a holomorphic map $z \mapsto A_{\theta}^{z}$ on the complex plane with values in $\mathcal{B}\left(H^{s}(M, E)\right)$ for any real number $s$, where $H^{s}(M, E)$ denotes the $H^{s}$-closure of the space $C^{\infty}(M, E)$ of smooth sections of $E$ (see, for example, [2]). The logarithm of $A$ is the bounded operator on $H^{s}(M, E)$, defined in terms of the derivative at $z=0$ of this complex power,

$$
\begin{aligned}
\log _{\theta} A & :=\left(\partial_{z} A_{\theta}^{z}\right)_{\left.\right|_{z=0}} \\
& =\frac{i}{2 \pi}\left(\partial_{z} \int_{\Gamma_{r, \theta}} \lambda_{\theta}^{z}(A-\lambda I)^{-z} d \lambda\right)_{\left.\right|_{z=0}} \\
& =\frac{i}{2 \pi} \int_{\Gamma_{r, \theta}} \log _{\theta} \lambda(A-\lambda I)^{-z} d \lambda,
\end{aligned}
$$

with the notation of (A.1).

The notion of logarithm extends to an admissible operator $A$ with positive order $a$ and spectral cut $\theta$ in the following way. For any positive $\epsilon$, the map $z \mapsto A_{\theta}^{z-\epsilon}$ of order $a(z-\epsilon)$ defines a holomorphic function on the half plane $\operatorname{Re}(z)<\epsilon$ with values in $\mathcal{B}\left(H^{s}(M, E)\right)$ for any real number $s$. Thus we can set

$$
\begin{aligned}
\log _{\theta} A & =A_{\theta}^{\epsilon}\left(\partial_{z}\left(A_{\theta}^{z}-\epsilon\right)\right)_{\left.\right|_{z=0}} \\
& =A_{\theta}^{\epsilon}\left(\partial_{z}\left(\frac{i}{2 \pi} \int_{\Gamma_{r, \theta}} \lambda_{\theta}^{z-\epsilon}(A-\lambda)^{-1} d \lambda\right)\right)_{\left.\right|_{z=0}} .
\end{aligned}
$$

For any positive $\epsilon$, the operator

$$
\log _{\theta} A A^{-\epsilon}=A^{-\epsilon} \log _{\theta} A
$$

lies in $\mathcal{B}\left(H^{s}(M, E)\right)$ for any real number $s$. It follows that $\log _{\theta} A$, which is clearly independent of the choice of $\epsilon>0$, defines a bounded linear operator from $H^{s}(M, E)$ to $H^{s-\epsilon}(M, E)$ for any positive $\epsilon$. We have

$$
\sigma\left(\log _{\theta} A\right)=a \log |\xi|+\sigma_{0}\left(\log _{\theta} A\right)
$$

where $\sigma_{0}\left(\log _{\theta} A\right)$ is a classical symbol whose asymptotic expansion

$$
\sigma_{0}\left(\log _{\theta} A\right) \sim \sum_{j=0}^{\infty} \sigma_{a-j, 0}\left(\log _{\theta} A\right)
$$

has homogeneous components of the form

$$
\sigma_{-j, 0}\left(\log _{\theta} A\right)(x, \xi)=|\xi|^{-j} \partial_{z}\left(\sigma\left(A_{\theta}^{z}\right)_{\alpha(z)-j}\left(x, \frac{\xi}{|\xi|}\right)\right)_{\left.\right|_{z=0}}
$$




\title{
References
}

[1] N. Berline, E. Getzler and M. Vergne, Heat Kernels and Dirac Operators, Grundlehren der Mathematischen Wissenschaften, 298 (Springer, Berlin, 1992).

[2] P. Gilkey, Invariance Theory, the Heat Equation and the Atiyah-Singer Index Theorem, 2nd edn, Studies in Advanced Mathematics (CRC Press, Boca Raton, FL, 1995).

[3] Ch. Kassel, 'Le résidu non commutatif (d'après M. Wodzicki)', Séminaire Bourbaki, Astérisque 177-178 (1989), 199-229.

[4] H. B. Lawson and M.-L. Michelson, Spin Geometry (Princeton University Press, Princeton, NJ, 1989).

[5] H. P. Mc Kean and I. M. Singer, 'Curvature and the eigenvalues of the Laplacian', J. Differential Geom. 1 (1967), 43-69.

[6] K. Okikiolu, 'The Campbell-Hausdorff theorem for elliptic operators and a related trace formula', Duke Math. J. 79 (1995), 687-722.

[7] K. Okikiolu, 'The multiplicative anomaly for determinants of elliptic operators', Duke Math. J. 79 (1995), 722-749.

[8] S. Paycha, 'Noncommutative formal Taylor expansions and second quantised regularised traces', in: Combinatorics and Physics, Clay Mathematics Institute Proceedings, to appear.

[9] S. Paycha and S. Scott, 'A Laurent expansion for regularised integrals of holomorphic symbols', Geom. Funct. Anal. 17 (2007), 491-536.

[10] S. Scott, 'Logarithmic structures and TQFT', Clay Math. Proc. 12 (2010), 309-331.

[11] S. Scott, Traces and Determinants of Pseudodifferential Operators, Math. Monographs (Oxford University Press, Oxford, 2009).

[12] S. Scott, 'The residue determinant', Comm. Partial Differential Equations 30 (2005), 483-507.

[13] R. T. Seeley, 'Complex powers of an elliptic operator, singular integrals', Proc. Symp. Pure Math., Chicago (American Mathematical Society, Providence, RI, 1966), pp. 288-307.

[14] M. Wodzicki, 'Spectral asymmetry and noncommutative residue' (in Russian) Thesis, (former) Steklov Institute, Sov. Acad. Sci., Moscow, New York 1984.

[15] M. Wodzicki, Noncommutative Residue. Chapter I. Fundamentals, Lecture Notes in Mathematics, 1289 (Springer, Berlin, 1987), pp. 320-399.

\author{
JOUKO MICKELSSON, Department of Mathematics and Statistics, \\ University of Helsinki, FI-00014 Helsinki, Finland \\ and \\ Department of Theoretical Physics, Royal Institute of Technology, \\ 10691 Stockholm, Sweden \\ e-mail: jouko@kth.se
}

SYLVIE PAYCHA, Laboratoire de Mathématiques, Complexe des Cézeaux, 63177 Aubière, France

e-mail: Sylvie.Paycha@math.univ-bpclermont.fr 